\title{
CONSTRUCTION METHODS FOR BHASKAR RAO AND RELATED DESIGNS
}

\author{
PETER B. GIBBONS ${ }^{\dagger}$ and RUDOLF MATHON ${ }^{\dagger \dagger}$
}

(Received 10 August 1984)

Communicated by W. D. Wallis

\begin{abstract}
Mathématical and computational techniques are described for constructing and enumerating generalized Bhaskar Rao designs (GBRD's). In particular, these methods are applied to $G B R D(k+$ $1, k, l(k-1) ; G)$ 's for $l \geqslant 1$. Properties of the enumerated designs, such as automorphism groups, resolutions and contracted designs, are tabulated. Also described are applications to group divisible designs, multi-dimensional Howell cubes, generalized Room squares, equidistant permutation arrays, and doubly resolvable two-fold triple systems.
\end{abstract}

1980 Mathematics subject classification (Amer. Math. Soc.): 05 B 05, 05 B 15, 05 B 25, 68 E 10.

\section{Introduction}

A balanced incomplete block design, $B I B D(\nu, b, r, k, \lambda)$ is an arrangement of $\nu$ elements into $b$ blocks such that (i) each element appears in exactly $r$ blocks; (ii) each block contains exactly $k(<\nu)$ elements, (iii) each pair of distinct elements appear together in exactly $\lambda$ blocks. Well known necessary conditions for a $B I B D(\nu, b, r, k, \lambda)$ to exist are $\nu r=b k$ and $\lambda(\nu-1)=r(k-1)$. Because of this dependence we shall use the abbreviated notation $B I B D(\nu, k, \lambda)$ to denote a $B I B D(\nu, b, r, k, \lambda)$. If in the definition of a $B I B D$ we do not require uniform block size (property (ii)), we obtain a so-called $(r, \lambda)$-design.

Two $B I B D(\nu, k, \lambda)$ 's $D_{1}$ and $D_{2}$ with element sets $V_{1}$ and $V_{2}$ respectively, are said to be isomorphic if there is a bijection $\theta: V_{1} \rightarrow V_{2}$ such that $\left\{x_{1}, \ldots, x_{k}\right\}$ is a

\footnotetext{
${ }^{\top}$ Work carried out while the author was visiting the University of Toronto, Canada.

${ }^{\dagger}$ Research reported by NSERC Grant No. A8651.

(c) 1987 Australian Mathematical Society $0263-6115 / 87 \$ A 2.00+0.00$
} 
block of $D_{1}$ if and only if $\left\{\theta\left(x_{1}\right), \ldots, \theta\left(x_{k}\right)\right\}$ is a block of $D_{2}$. An automorphism of a $B I B D$ is an isomorphism of the $B I B D$ with itself. The set of all automorphisms, under the usual composition of mappings, forms the automorphism group of the $B I B D$.

A generalized Bhaskar Rao design is defined as follows. Let $W$ be a $\nu \times b$ matrix with elements from $G \cup\{0\}$, where $G=\left\{h_{1}=e, h_{2}, \ldots, h_{g}\right\}$ is a finite group of order $g$. Then $W$ can be expressed as a sum $W=h_{1} A_{1}+\cdots+h_{g} A_{g}$, where $A_{1}, \ldots, A_{g}$ are $\nu \times b(0,1)$-matrices such that the Hadamard product $A_{i}^{*} A_{j}=0$ for any $i \neq j$. Denote by $W^{+}$the transpose of $h_{1}^{-1} A_{1}+\cdots+h_{g}^{-1} A_{g}$ and let $N=A_{1}+\cdots+A_{g}$. Then $W$ is a generalized Bhaskar Rao design denoted by $G B R D(\nu, b, r, k, \lambda ; G)$ if

(i) $W W^{+}=r e I+\lambda / g\left(h_{1}+\cdots+h_{g}\right)(J-I)$,

(ii) $N N^{T}=(r-\lambda) I+\lambda J$.

The second condition merely prescribes that $N$ be the incidence matrix of a $B I B D(\nu, b, r, k, \lambda)$. Because of the parameter dependencies for $B I B D$ mentioned above we shall use the shorter notation $\operatorname{GBRD}(\nu, k, \lambda ; G)$ for a generalized Bhaskar Rao design.

A $G B R D(\nu, k, \lambda ; G)$ with $\nu=b$ is a symmetric $G B R D$ or generalized weighing matrix. If $W$ has no 0 entries then the $G B R D$ is also known as a generalized Hadamard matrix.

A group-divisible design, $G D D(\nu \times g, k, \lambda)$ is an incidence structure $(X, B)$ consisting of a set $X,|X|=\nu g$, partitioned into $\nu$ disjoint $g$-subsets (groups), $X=X_{1} \cup \cdots \cup X_{v}$, and a collection $B$ of $k$-subsets of $X$ (blocks) such that

(i) each point $x \in X$ is incident with $r$ blocks,

(ii) $\left|L \cap X_{i}\right| \leqslant 1$ for every block $L \in B$ and $i=1, \ldots, \nu$,

(iii) if $x \in X_{i}, y \in X_{j}, i \neq j$, there are exactly $\lambda$ blocks incident with $x$ and $y$.

If $|B|=b g$, then $b k=r \nu$, and $\lambda g(v-1)=r(k-1)$. A $G D D(\nu \times g, k, \lambda)$ with $g=1$ is a $B I B D(\nu, k, \lambda)$. GDD isomorphism is defined in the same way as $B I B D$ isomorphism.

From a $G B R D(\nu, k, \lambda ; G),|G|=g$, we can form a $G D D(\nu \times g, k, \lambda / g)$ as follows. For any $h \in G$ let $P_{h}$ denote the corresponding $g \times g$ permutation matrix, $P_{h_{1}}+\cdots+P_{h_{g}}=J$. If $W$ is the $\nu \times b$ matrix of a GBRD let $N$ be the $\nu g \times b g(0,1)$-matrix obtained from $W$ by replacing any group element $h$ by $P_{h}$ and any 0 entry by a $g \times g$ all-zero matrix. Then $N$ is the incidence matrix of a $G D D(\nu \times g, k, \lambda / g)$.

Two $G B R D(\nu, k, \lambda ; G)$ 's $W$ and $W^{\prime}$ are isomorphic if there exist two $G$-permutation matrices $P$ and $Q$, and an automorphism $\sigma$ of the group $G$ such that $W=\operatorname{Po}(W) Q$. The isomorphism itself will be denoted by the triple $(P, \sigma, Q)$. It is probably instructive at this point to see how such an isomorphism preserves the $G B R D$ property that for any two rows $w_{i}^{\prime}, w_{j}^{\prime}(i \neq j)$ of $W^{\prime}, w_{i}^{\prime} *\left(w_{j}^{\prime}\right)^{-1}=$ $\lambda / g\left(h_{1}, \ldots, h_{g}\right)$. Clearly, the rearrangement of rows and columns of $W$ preserves 
the property, so let us concentrate on the effect of applying the automorphism $\sigma$ to each entry of $W$, and then pre-multiplying each row $i$ by $x_{i} \in G$, and post-multiplying each column $h$ by $y_{h} \in G$. For $i \neq j$ we have

$$
\begin{aligned}
w_{i}^{\prime} *\left(w_{j}^{\prime}\right)^{-1} & =\sum_{h=1}^{b} x_{i} \sigma\left(w_{i h}\right) y_{h} *\left(x_{j} \sigma\left(w_{j h}\right) y_{h}\right)^{-1} \\
& =\sum_{h=1}^{b} x_{i} \sigma\left(w_{i h}\right) y_{h} * y_{h}^{-1}\left(\sigma\left(w_{j h}\right)\right)^{-1} x_{j}^{-1} \\
& =\sum_{h=1}^{b} x_{i} \sigma\left(w_{i h}\right) \sigma\left(w_{j h}^{-1}\right) x_{j}^{-1} \\
& =x_{i} \sigma\left(\sum_{h=1}^{b} w_{i h} w_{j h}^{-1}\right) x_{j}^{-1} \\
& =x_{i} \sigma\left(\lambda / g\left(h_{1}+\cdots+h_{g}\right)\right) x_{j}^{-1} \\
& =x_{i}\left(\lambda / g\left(h_{1}+\cdots+h_{g}\right)\right) x_{j}^{-1} \\
& =\lambda / g\left(h_{1}+\cdots+h_{g}\right) .
\end{aligned}
$$

The operations carried out in the above proof will be applied extensively in the isomorph rejection procedures to be discussed in Section 3.

An isomorphism of $W$ with itself is called an automorphism of $W$. The set of automorphisms of a GBRD form a group $\Gamma$ under the operations of matrix multiplication and mapping composition, i.e., if $\left(P_{1}, \sigma_{1}, Q_{1}\right)$ and $\left(P_{2}, \sigma_{2}, Q_{2}\right)$ are two automorphisms, then so is $\left(P_{1}, P_{2}, \sigma_{1} \sigma_{2}, Q_{1} Q_{2}\right)$. Note that $\Gamma$ contains a subgroup isomorphic to $G$, since for any $h \in G,\left(D_{h}, \sigma_{h}, D_{h^{-1}}\right) \in \Gamma$, where $\sigma_{h}(w)$ is the inner automorphism $h^{-1} w h$ of $G$, and $D_{h}$ is an $h$-diagonal matrix.

We note that every automorphism of a GBRD is an automorphism of the underlying $G D D$, but that the converse is not true in general.

A design $(X, B)$ (which can be a $B I B D$, a $G D D$, or an $(r, \lambda)$-design) is said to be resolvable if there exists a partition $R$ of the set of blocks $B$ into subsets $R_{1}, \ldots, R_{u}$, called parallel classes, such that each $R_{i}$ is a partition of $X$. Two resolutions $R=\left\{R_{1}, \ldots, R_{u}\right\}, R^{\prime}=\left\{R_{1}^{\prime}, \ldots, R_{u}^{\prime}\right\}$ of a design $(X, B)$ are orthogonal if $\left|R_{i} \cap R_{j}^{\prime}\right| \leqslant 1$ for all $i, j=1, \ldots, u$. A design with two orthogonal resolutions is called doubly resolvable.

Bhaskar Rao designs have been studied by a number of authors. For example, Bhaskar Rao [1], Street and Rodger [25], and Seberry [22] have examined such designs in connection with the construction of partially balanced block designs. Generalized Hadamard designs have been studied by Butson [2,3], and by Shirkhande [24] in connection with combinatorial designs, by Delsarte and Goethals [5] in connection with codes, and by Drake [7] in connection with 
$\lambda$-geometries. Generalized weighing matrices were first introduced by Yates [29] in connection with determining the accuracy of measurements. Since then they have been studied extensively $[8,9,13,26,27]$.

In this paper we shall study GBRD's based on $l$-multiples $(l \geqslant 1)$ of the unique $B I B D$ with blocksize $k, \nu=k+1$ and $\lambda=k-1$ (i.e. the complement of the complete $B I B D(\nu, 1,0))$. A necessary condition for the existence of a $G B R D(k+$ $1, k, l k-l ; G)$ is that $g=|G|$ divides $l(k-1)$.

\section{Existence}

Two infinite families of $G B R D(k+1, k, l k-l ; G)$ 's are known to exist. One arises from cyclotomic classes in finite fields, while the second can be constructed from projective planes containing a Baer subplane. In this section we describe the direct constructions which produce these families. We conclude the section with two methods for constructing new GBRD's from old ones. These latter constructions are described in the form of Theorems 1 and 2.

\section{Construction 1}

This construction produces generalized weighing matrices and corresponds to the case $l=1[9,14,16,23]$.

Let $q=m t+1$ be a prime-power and let $\alpha$ be a primitive root in the finite field $G F(q)$ with elements $a_{1}, \ldots, a_{q}$. Consider the partition of $G F(q)$ into $m+1$ so-called cyclotomic classes $C_{0}, C_{1}, \ldots, C_{m}$ defined as follows:

$$
C_{0}=\{0\}, C_{i+1}=\left\{\alpha^{k j+i} \mid j=0,1, \ldots, t-1\right\}, \quad i=0,1, \ldots, m-1 .
$$

Let $A$ be the $q \times q$ symmetric matrix with a zero diagonal and off-diagonal entries $w_{i j}=h_{s}$ if and only if $a_{i}-a_{j} \in C_{s}$ for some $s, 1 \leqslant s \leqslant m, 1 \leqslant i, j \leqslant q$. Then the matrix $W=\left[\begin{array}{cc}0 & e^{T} \\ e & A\end{array}\right], e^{T}=(1, \ldots, 1)$ is a $\operatorname{GBRD}(q+1, q, q-1 ; G)$, where $G=\left\{h_{1}, \ldots, h_{m}\right\}$ is a cyclic group of order $m, h_{i}=\beta^{i-1}, i=1, \ldots, m, \beta^{m}=\beta^{0}$ $=1$.

We make a few observations.

1. If $m=2$ and $G=\{1,-1\}$ under multiplication, then the matrix $W$ of a $G B R D(k+1, k, k-1 ; G)$ is called a conference matrix. Several other constructions are known for conference matrices yielding other families of GBRD's (see $[12,15])$.

2. If $q=r^{2}$ is a square and $m=r+1$, then this construction can be extended to any group $G$ of order $m$, since $A$ corresponds to an affine plane of order $r$.

3. A family of $G B R D(k+1, k, k-1 ; G)$ 's with $k$ not a prime-power can be constructed from so-called pseudo-cyclic association schemes $[14,16]$. Here $k=$ $2^{m-1}\left(2^{m}-1\right)$ and $G$ is a cyclic group of order $g=2^{m-1}, m \geqslant 3$. So, for example, 
if $m=3$ we obtain a $G B R D(29,28,27 ; G)$ (see [16]):

$$
W=\left[\begin{array}{ll}
0 & e^{T} \\
e & A
\end{array}\right], \quad A=\left[\begin{array}{llll}
0 & e^{T} & \omega e^{T} & \bar{\omega} e^{T} \\
e & A & B & C \\
\omega e & B & D & E \\
\bar{\omega} e & C & E & F
\end{array}\right], \quad G=\{1, \omega, \bar{\omega}\},
$$

where $A, \ldots, F$ are the symmetric circulant matrices

$$
\begin{array}{ll}
A=(01 \omega \omega \bar{\omega} \bar{\omega} \omega 1) & D=(0 \bar{\omega} 1 \bar{\omega} \omega \omega \bar{\omega} 1 \bar{\omega}) \\
B=(\bar{\omega} \bar{\omega} 1 \omega 11 \omega 1 \bar{\omega}) & E=(1 \omega \omega \bar{\omega} 11 \bar{\omega} \omega \omega) \\
C=(\omega \bar{\omega} \omega 1 \bar{\omega} 1 \omega \bar{\omega}) & F=(0 \omega \bar{\omega} 1111 \bar{\omega} \omega)
\end{array}
$$

and $\omega$ is a cube-root of unity, $\bar{\omega}=\omega^{2}$.

\section{Construction 2}

A family of $G B R D(k+1, k, l k-1 ; G)$ 's with $l=k$ can be constructed from certain projective planes $[22,28]$.

Let $I I$ be a projective plane of order $q^{2}$ and let $\Pi^{\prime} \subset \Pi$ be a Baer subplane of order $q$, where $q$ is a prime power. Given a point $P$ in $\Pi^{\prime}$, there are exactly $q+1$ lines $L_{1}, \ldots, L_{q+1}$ in $\Pi$ incident with $P$ which intersect $\Pi^{\prime}$ in $q+1$ points. Let $X_{i}$ be the set of $q^{2}-q$ points of $L_{i}$ not in $\Pi^{\prime}, i=1, \ldots, q+1$. Since $\Pi^{\prime}$ is a Baer subplane every line of $\Pi$ is incident with $q+1$ or 1 point of $\Pi^{\prime}$. For every point $Q_{j} \neq P$ of $\Pi^{\prime}$ let $B_{j}$ be the set of $q^{2}-q$ lines of $\Pi$ incident with the single point $Q_{j}$ of $\Pi^{\prime}, j=1,2, \ldots, q^{2}+q$. Then $(X, B)$ is a $G D D\left((q+1) \times\left(q^{2}-\right.\right.$ $q), q, 1)$, where $X=X_{1} \cup \cdots \cup X_{q+1}, B=B_{1} \cup \cdots \cup B_{q^{2}+q}$. If $I I$ is a translation plane then the partitions of $X$ and $B$ induce a $G B R D\left(q+1, q, q^{2}-q ; G\right)$ where $G$ is a nonabelian group of order $q^{2}-q$. This group is isomorphic to the semi-direct product of the multiplicative and additive groups of $G F(q)$ generated by the transformations of the form $a x+b, a, b \in G F(q), a \neq 0$.

Again, let us make a few comments.

1. Any $G D D$ given by Construction 2 is highly resolvable. In fact, in such a $G D D$ there exists a set of $q^{2}-q$ mutually orthogonal resolutions. To see this let $S$ be the set of points in $\Pi$ not incident with any of the lines $L_{1}, \ldots, L_{q+1}$, and let $K$ be the set of lines in $\Pi$ incident with $P$ and no other point of $\Pi^{\prime}$. Clearly, $|S|=q^{4}-q^{3},|K|=q^{2}-q$, and every line in $K$ is incident with exactly $q^{2}$ points of $S$, say $S_{1}, \ldots, S_{q^{2}}$. Since $\Pi$ is a projective plane, the blocks $B_{i}^{\prime} \subseteq B$ incident with $S_{i}$ (restricted to $X$ ) form a parallel class, and $B_{1}^{\prime} \cup \cdots \cup B_{q^{2}}^{\prime}=B$, $\left|B_{i}^{\prime} \cap B_{j}^{\prime}\right|=0, i \neq j$, form a resolution of the $G D D$. Moreover, two distinct lines of $K$ induce two resolutions which are mutually orthogonal. Conversely, given a $G D D\left((q+1) \times\left(q^{2}-q\right), q, 1\right)$ together with $q^{2}-q$ mutually orthogonal resolutions we can reconstruct $\Pi$ and $\Pi^{\prime}$. 


\begin{tabular}{|l|c|c|c|c|c|c|c|}
\hline \multicolumn{7}{|c|}{ Numbers and properties of generated Bhaskar Rao designs } \\
\hline Parameters & Design & $|G|$ & Rep. blocks & $D$ & $\|$ classes & Resolutions & Contractions \\
\hline \hline $6,5,4 ; Z_{4}$ & $B$ & 480 & $1^{6}$ & & & & \\
\hline $6,5,4 ; Z_{2}^{2}$ & - & & & & & & \\
\hline $6,5,8 ; Z_{8}$ & - & & & & & & \\
\hline $6,5,8 ; D_{4}$ & - & & & & & & \\
\hline $6,5,8 ;$ & $C$ & 96 & $1^{12}$ & & & & $\Psi, \Phi_{1}, \Omega_{1,2,3}$ \\
$Z_{2} \times Z_{4}$ & & & & & & & \\
\hline $6,5,8 ; Z_{2}^{3}$ & $D_{1}$ & 192 & $1^{12}$ & & & & $\Phi_{2}, \Phi_{3}, \Omega_{1}, \Omega_{2}$ \\
& $D_{2}$ & 192 & $1^{12}$ & & & & $\Phi_{3}, \Phi_{4}, \Omega_{2}, \Omega_{4}$ \\
\hline $6,5,8 ; Q$ & $E_{1}$ & 48 & $1^{12}$ & & & & $\Phi_{3}, \Omega_{2}$ \\
& $E_{2}$ & 48 & $1^{12}$ & & & & $\Phi_{3}, \Omega_{2}$ \\
\hline $6,5,8 ; Z_{4}^{\dagger}$ & $\Psi$ & 24 & $1^{12}$ & & & & $\Omega_{1}$ \\
\hline $6,5,8 ; Z_{2}^{2 \dagger}$ & $\Phi_{1}$ & 48 & $1^{12}$ & & & & $\Omega_{1}, \Omega_{2}, \Omega_{3}$ \\
& $\Phi_{2}$ & 32 & $1^{12}$ & & & & $\Omega_{1}, \Omega_{2}$ \\
& $\Phi_{3}$ & 24 & $1^{12}$ & & & & $\Omega_{2}$ \\
& $\Phi_{4}$ & 32 & $1^{12}$ & & & & $\Omega_{2}, \Omega_{4}$ \\
\hline $6,5,8 ; Z_{2}^{* \dagger}$ & $\Omega_{1}$ & 96 & $1^{12}$ & & & & \\
& $\Omega_{2}$ & 24 & $1^{12}$ & & & & \\
& $\Omega_{3}$ & 48 & $1^{12}$ & $d$ & & & \\
& $\Omega_{4}$ & 96 & $1^{12}$ & $d$ & & & \\
\hline
\end{tabular}

${ }^{\dagger}$ Not a complete enumeration-designs are contractions of other designs.

*t The total number of these designs is 19

Parts of this table require some explanation. Firstly, the repeated block structure of a GBRD is indicated using the notation $a_{1}^{b_{1}}, \ldots, a_{l}^{b_{l}}$. This means that $b_{i}$ blocks are repeated $a_{i}$ times, $1 \leqslant i \leqslant l$. In the column labelled $D$, a $d$ indicates the design is decomposable. The actual decompositions are displayed in the appendices. Also in the table the numbers of distinct (rather than non-isomorphic) parallel classes and resolutions of the underlying $G D D$ 's are displayed. The normal subgroups used to produce the listed contractions are detailed in the table on the opposite page.

Before moving on to discuss various interesting structures which arise from these generated designs, let us first briefly explain how some of their properties were determined. Recall that our generation procedure does not incorporate a complete isomorph rejection procedure. To partition the output designs into their isomorphism classes we therefore applied the $B I B D$ isomorphism procedure described in $[10,11]$ to the underlying $G D D$ 's. A strong invariant for this purpose was provided by a clique analysis of the designs, omitting intersections among blocks belonging to the same block orbit. 


\begin{tabular}{|l|l|l|l|}
\hline \multicolumn{3}{|c|}{ Derivation of Contracted Designs } \\
\hline Group & Parent Design & Normal Subgroup(s) & Contracted Design(s) \\
\hline \hline$Z_{4}$ & Any & $\{1, \beta\}$ & As above \\
\hline$Z_{6}$ & Any & $\{1, \beta, \delta\}$ & As above \\
& Any & $\{1, \gamma\}$ & As above \\
\hline$S_{3}$ & Any & $\{1, \alpha, \beta\}$ & As above \\
\hline$Z_{2} \times Z_{4}$ & $C$ & $\{1, \delta\},\{1, \beta\}$ & $\Psi, \phi_{1}$ \\
\hline$Z_{2}^{3}$ & $D_{1}$ & $\{1, \alpha\},\{1, \delta\}$ & $\Phi_{2}, \Phi_{3}$ \\
& $D_{2}$ & $\{1, \delta\},\{1, \alpha\}$ & $\Phi_{3}, \Phi_{4}$ \\
\hline$Q$ & $E_{1}, E_{2}$ & $\{1, \delta\}$ & $\Phi_{3}$ \\
\hline$Z_{2}^{2}$ & $F$ & $\{1, \alpha\}$ & $B_{3}$ \\
& $\Phi_{1}$ & $\{1, \alpha\},\{1, \beta\},\{1, \gamma\}$ & $\Omega_{1}, \Omega_{2}, \Omega_{3}$ \\
& $\Phi_{2}$ & $\{1, \alpha\},\{1, \beta\}$ & $\Omega_{1}, \Omega_{2}$ \\
& $\Phi_{3}$ & $\{1, \alpha\}$ & $\Omega_{2}$ \\
& $\Phi_{4}$ & $\{1, \alpha\},\{1, \beta\}$ & $\Omega_{4}, \Omega_{2}$ \\
\hline
\end{tabular}

${ }^{\dagger}$ Only those that produce non-isomorphic designs.

The automorphism groups of the GBRD's were similarly obtained by applying the $B I B D$ automorphism group generator program of $[10,11]$ to the underlying $G D D$ 's. Care, of course, needs to be taken to ensure that we only consider automorphisms which preserve block orbits. However, in only one case, the $G B R D\left(4,3,6 ; Z_{6}\right) G_{5}$, was the group of the $G B R D$ different from that of the underlying $G D D$. In this case the group orders are 144 and 1296 respectively.

Note that a $\operatorname{GBR} D\left(\nu, k, \lambda ; G_{1}\right)$ and a $\operatorname{GBR} D\left(\nu, k, \lambda ; G_{2}\right)$ over different groups $G_{1}$ and $G_{2}$ of the same order $g$ may produce isomorphic $G D D(v \times g, k, \lambda)$ 's. Two examples of this are the $G D D$ 's arising from $G_{9}$ and $G_{10}$ over $Z_{6}$. These are isomorphic to the $G D D$ 's arising from $\mathrm{H}_{5}$ and $\mathrm{H}_{3}$, respectively, over $\mathrm{S}_{3}$.

In determining the numbers of parallel classes and resolutions of a $G D D$, a clique-finding program was again of some help. Suppose we have a $G D D(\nu, k, \lambda)$ $D$ with $b$ blocks, where $\nu / k=u$ and $b / u=w$. Form the block intersection graph $G_{B}$ in which the vertices represent the blocks of $D$, and in which vertices $i$ and $j$ are adjacent if and only if blocks $i$ and $j$ are disjoint. Then a $u$-clique in $G_{B}$ corresponds to a parallel class in $D$.

Having obtained all $u$-cliques in $G_{B}$ we now form the parallel class intersection $G_{P}$ in which the vertices represent the set of parallel classes, with two vertices adjacent if and only if the associated parallel classes are disjoint. A $w$-clique in $G_{P}$ corresponds to a resolution of $D$. 
2. Applying Construction 2 to the translation plane of order 9 , or to its dual, yields a $G B R D\left(4,3,6 ; S_{3}\right)$. It is listed in the Appendix as design $H_{1}$. The blocks of the corresponding $G D D(4 \times 6,3,1)$ are resolvable in $t=6$ mutually orthogonal ways. This yields a 6 -dimensional generalized Howell cube of side $r=9$, order $\nu=24$, and uniform block size $k=3$, achieving a conjectured upper bound of $t=r-k$ on the dimension $t$ of such a cube. We note that every pair of elements is contained in at most one block of the cube (see Rosa [21]).

We now describe two methods for constructing new GBRD's from old ones. The first method can be used to construct a large number of $G B R D$ 's with $l=k$.

THEOREM 1. Suppose there exists a $G B R D(k+1, k, k-1 ; G)$ and $a$ $G B R D(k, k, k ; H)$ for some $k>1$. Then there exists a $G B R D\left(k+1, k, k^{2}-k\right.$; $G \times H)$, where $G \times H$ is the direct product of $G$ and $H$.

ProOF. Form a matrix $W^{\prime}$ by taking $k$ identical copies of $W$, a $G B R D(k+$ $1, k, k-1 ; G)$, and denote by $W_{i}^{\prime}$ the $k$ columns of $W^{\prime}$ containing a 0 in the $i$ th position. Subscript the $k \times k$ non-zero submatrix of $W_{i}^{\prime}$ by the entries of $V$, a $G B R D(k, k, k ; H)$, for $i=1,2, \ldots, k+1$. From the properties of $W$ and $V$ it follows that the resulting matrix with entries $g_{h}, g \in G, h \in H$ forms a $G B R D(k$ $\left.+1, k, k^{2}-k ; G \times H\right)$ if the product $g_{h} g_{h}^{\prime}$ is interpreted as $\left(g g^{\prime}\right)_{h h}$. We note that the designs $V$ used to subscript $W_{i}^{\prime}$ do not have to be identical or even isomorphic.

If $k=q$ is a prime power then a generalized Hadamard matrix corresponding to a $\operatorname{GBRD}(q, q, q ; H)$ can be constructed from an affine plane of order $q$, and a $G B R D(q+1, q, q-1 ; G)$ is given by Construction 1 . For $q=3$ this yields the $G B R D\left(4,3,6 ; Z_{6}\right) G_{5}$ listed in the appendices.

We conclude this section with another method for constructing new GBRD's from old ones.

THEOREM 2. Let $W$ be a $G B R D(\nu, k, \lambda ; G)$ and suppose that $G$ contains a normal subgroup $T$. Then there exists a $G B R D(\nu, k, \lambda ; H)$, where $H=G / T$ is the factor group of $G$ with respect to $T$.

Proof. Use the homomorphism from $G$ to $H$ with kernel $T$ to obtain $W^{\prime}$ from $W$. It is easily verified that $W^{\prime}$ is a $G B R D$.

The new GBRD $W^{\prime}$ will be called a contraction of $W$ with respect to $T$. We note that choosing different normal subgroups of the same order in $W$ may lead to non-isomorphic contractions $W^{\prime}$ (see, for example, the $\operatorname{GBRD}\left(6,5,8 ; Z_{2} \times Z_{4}\right.$ ), $C$ in the appendices). 


\section{Enumeration}

In this section we shall describe a computational method for enumerating non-isomorphic GBRD's with fixed parameters $\nu, k, \lambda$ and $G$. This method was implemented in the programming language Algol $W$ on an IBM 3033 computer, and was used to enumerate the family of $G B R D(k+1, k, l(k-1)$; $G)$ 's listed in the appendices.

Recall from Section 1 that any $G B R D(\nu, k, \lambda ; G)$ is based on the incidence matrix $N$ of a $B I B D(\nu, k, \lambda)$. A necessary condition for two $G B R D$ 's to be isomorphic is that their underlying $B I B D$ 's be isomorphic. A first step, then, in our procedure for enumerating $G B R D$ 's is to enumerate the underlying $B I B D$ 's using techniques such as those described by Gibbons, Mathon and Corneil [10, 11]. Then our $G B R D$ enumeration algorithm accepts $N$, in addition to the parameters $\nu, k, \lambda$ and $G$, and enumerates all $G B R D$ 's with this parameter set.

In the case of a $G B R D(k+1, k, l(k-1) ; G)$ the underlying $B I B D(k+$ $1, k, l(k-1))$ is unique up to isomorphism. The copy of $N$ used by our algorithm must be permuted so that identical columns (corresponding to repeated blocks) are grouped together to form $\mathrm{N}$-cells. This cell structure is used to implement isomorph rejection procedures which we shall describe shortly.

The construction algorithm is a 2-level backtrack procedure based on the idea of an orderly algorithm as introduced by Read [19]. We begin by defining an ordering $h_{1}<h_{2}<\cdots<h_{g}$ on the elements of $G$, and then proceed to construct $W$ row by row, replacing the " 1 " entries of $N$ by elements from the group $G$ subject to the constraint $\sum_{l=1}^{b} w_{i l}\left(w_{j l}\right)^{-1}=\lambda / g\left(h_{1}+\cdots+h_{g}\right)$ (for $i \neq j$ ). Individual rows are considered in strictly increasing lexicographical order, with the result that any completed matrices will also be output in increasing order.

This simple algorithm, as it stands, would be impractical for most problems unless some form of isomorph rejection procedures were implemented. For this purpose let us define the canonical representative of a class $C$ of GBRD's based on $N$ as the minimum design in $C$. We would like our algorithm to generate only designs which are canonical representatives of their isomorphism classes.

To accomplish this we rely on the fact that the output GBRD's are produced in increasing lexicographical order. To each generated design $D$ we apply a minimization operation $m$ which transforms $D$ to an isomorphic (and hopefully smaller) design $m(D)$. If $m(D)<D$, then $D$ can be rejected since it is isomorphic to a design considered earlier in the search. This rejection check can also be applied to partially completed designs. In fact such early checks are crucial in developing an effective enumeration algorithm.

If $m(D)$ turns out to be the canonical representative of $D$ 's isomorphism class, then we have a complete isomorph rejection procedure, i.e. our algorithm will produce only canonical representatives from the set of isomorphism classes. For 
various practical reasons we stopped a little short of implementing such a complete rejection procedure, while still maintaining an effective rejection rate.

Suppose that the matrix $N$ has $N$-cells $c_{i}, i=1, \ldots, l$, where $n_{m h}=n_{m j}$ for $h, j \in c_{i}$ and $m=1, \ldots, \nu$, and suppose that we have constructed $W^{t}$, the first $t$ rows $(0 \leqslant t<\nu)$ of $W$. We define the set $F_{t+1}=\left\{f_{i}: i=1, \ldots, m\right\}$ of $W$-cells as follows: $i, j \in f_{h}$ if and only if $i, j \in c_{p}$ for some $1 \leqslant p \leqslant l$, and $w_{m i}=w_{m j}$ for $1 \leqslant m \leqslant t$. That is, $W$-cells correspond to consecutive (partially completed) repeated blocks in the $G B R D$ under construction. Note that the $W$-cell partitioning is a refinement of the $N$-cell partitioning.

We observe now that in constructing row $t+1$, for any $i, j \in f_{h}(i<j)$ we can stipulate $w_{t+1, i} \leqslant w_{t+1, j}$. For if $w_{t+1, i}>w_{t+1, j}$ we could swap columns $i$ and $j$ in $W^{t+1}$ to obtain an isomorphic partial configuration strictly less than the current one. In other words, when constructing row $t+1$, group elements in columns belonging to the same $W$-cell are placed in non-decreasing order.

Our second observation concerns a non-zero element $w_{i j}$ for which either $n_{i h}=0$ for $1 \leqslant h<j$, or $n_{h j}=0$ for $1 \leqslant h<i$. Such a row (respectively, column) header can be set equal to $e$, since if we set it to $x(\neq e)$ we can pre( post-) multiply the row (column) of $W$ by $x^{-1}$ to produce an isomorphic matrix $W^{\prime}<W$.

The third check carried out is more involved. Let $H$ be the automorphism group of $N$ and let $H_{t}$ be the subgroup of $H$ which permutes the first $t$ rows among themselves. Actually, in $H$ we shall omit consideration of reorderings of columns within cells. The implications of this simplification will become apparent later.

Also let $A$ be the automorphism group of the given group $G$, and define a minimization operation $m$ as follows. Suppose $W^{t}$ represents the first $t$ rows of $W$. Then $m\left(W^{t}\right)$ is formed from $W^{t}$ as follows:

(i) Scan $W^{t}$ row by row, from left to right. For each row (column) header $w_{i j}=x$, reduce $w_{i j}$ to $e$ by pre- (post-) multiplying row $i$ (column $j$ ) by $x^{-1}$.

(ii) Sort the columns of each $N$-cell in the resulting matrix into non-decreasing order.

Now suppose our algorithm has constructed $W^{t}$. Then this partial configuration may be rejected, forcing a backtrack, if there exists $\sigma \in A, \varphi \in H_{t}$ such that $m\left(\varphi\left(\sigma\left(W^{t}\right)\right)\right)<W^{t}$. In terms of our algorithm, this means checking every $\sigma \in A$ and every $\varphi \in H_{t}$ to see whether $m\left(\varphi\left(\sigma\left(W^{t}\right)\right)\right)<W^{t}$.

This turns out to be an effective isomorph rejection procedure. However, it is not complete, for the following reasons. Suppose, just before the minimization step described above, we have a matrix $\left(W^{\prime}\right)^{t}$ in which there is a row header $w_{i j}^{\prime}$ $(1 \leqslant i \leqslant t)$ which belongs to a $W$-cell $f \in F^{i}$. To obtain true minimal form we must in turn reduce each entry in $f$ to $e$, sort entries within the same $W$-cell, and 
select the reduction that produces the smallest row $i$. In the case that all row headers belong to cells of size 1 , our rejection procedure is complete. However, because of its complexity we decided against implementing the full check for row header cells of size $>1$.

Results from the application of this algorithm are described in the following section, and also in the appendices

We conclude this section by mentioning briefly an extension of this eumerative technique to the construction of $B I B D$ 's which contain a subgroup of the automorphism group of a specified type.

Suppose we are interested in enumerating all $B I B D(\nu, b, r, k, \lambda)$ 's containing a subgroup $G$ of order $g$ of the automorphism group. Let us represent the group $G$ additively, and assume that $g \mid \nu$ and $g \mid b$. Then in the designs we are looking for, the $\nu$ elements partition into $\nu / g(=n$, say) orbits of size $g$ under the action of $G$. In fact each such design can be represented by $s(=b / g)$ base blocks and can be generated by applying the elements of $G$ to these base blocks. Our task will be to construct this set of base blocks.

We begin by considering the $n \times s$ intersection matrix $N$, where each element $n_{i j}$ is defined as the number of elements from orbit $i$ contained in base block $j$, $1 \leqslant i \leqslant n, 1 \leqslant j \leqslant s . N$ must satisfy the following constraints.

(i) $\sum_{i=1}^{n} n_{i j}=k, 1 \leqslant j \leqslant s$, i.e. each block must contain $k$ elements.

(ii) $\sum_{j=1}^{s} n_{i j}=r, 1 \leqslant i \leqslant n$, i.e. each element occurs in $r$ blocks.

(iii) $\sum_{l=1}^{s} n_{i l}^{2}-n_{i l}=\lambda(g-1), 1 \leqslant i \leqslant n$, i.e. each pair of elements from the same orbit (or pure pair) must occur together in exactly $\lambda$ blocks.

(iv) $\sum_{l=1}^{s} n_{i l} n_{j l}=\lambda g, 1 \leqslant i<j \leqslant n$, i.e. each pair of elements from different orbits (or mixed pair) must occur together in exactly $\lambda$ blocks.

A necessary condition for two of the $B I B D(\nu, b, r, k, \lambda)$ 's under consideration to be isomorphic is that the underlying $N$ matrices be isomorphic. We therefore commence our enumeration by generating all non-isomorphic such $N$ matrices with the given constraints. This can be done by adapting the backtrack and isomorph rejection techniques described earlier in this section. The larger the group $G$ is, the smaller the matrix $N$ will be in relation to the incidence matrix of the $B I B D$. In many cases, with a non-trivial group $G$, we can carry out perfect isomorph rejection techniques at this stage. However, in the extreme case where $g=1$ (i.e. where we assume only the existence of the identity automorphism), the matrix $N$ simply coresponds to the incidence matrix of a $B I B D(\nu, b, r, k, \lambda)$ so that our enumeration algorithm will produce all $B I B D$ 's with these parameters.

In the second stage of the enumeration we input each $N$ matrix into a generalization of the algorithm described at the beginning of this section. The main difference here is that the entries in $N$ are not restricted simply to having the values 0 or 1 . Instead each entry $n_{i j}$ satisfies the constraint $0 \leqslant n_{i j} \leqslant k$. Our task is to 'fill' each entry $n_{i j}$ with $n_{i j}$ elements from group $i$, subject to the 
constraint that all pure and mixed differences are covered exactly $\lambda$ times. As with our previous algorithm, isomorph rejection can be achieved by applying elements from the automorphism group of $N$, in combination with permutations from the group $G$, to each generated matrix $W$. We are careful to order the group elements filling each entry $n_{i j}$ both during construction and after applying isomorphisms.

The orbit structure mentioned above can be generalized in many ways. For example, take the $\operatorname{BIBD}(45,99,11,5,1)$ and assume the existence of a (necessarily cylic) group $G$ of order 11 . Then under the action of $G$ the 45 elements partition into a fixed element and 4 orbits of size 11 . Our intersection matrix in this case represents the structure of 8 base blocks of size 5 and 1 base block of size 4 (omitting the fixed element).

Using the described algorithm we first generated a total of 13 non-isomorphic intersection matrices with this structure. However, none of these matrices could be used to produce a complete design with the prescribed group structure. The conclusion, then, is that there exists no $\operatorname{BIBD}(45,99,11,5,1)$ with an automorphism of order 11 .

This method has been applied successfully to the search for $B I B D(45,99,11,5$, 1 )'s with other prescribed group structures, as well as to other designs.

\section{Analysis}

Various sets of $G B R D(k+1, k, l k-l ; G)$ 's with $l \geqslant 1$ were obtained using the methods described in Section 3. The exact numbers and properties of these designs are displayed in the following table. The designs themselves are listed in the appendices.

\begin{tabular}{|c|l|r|c|c|c|c|c|}
\hline \multicolumn{8}{|c|}{ Numbers and properties of generated Bhaskar Rao designs } \\
\hline Parameters & Design & $|G|$ & Rep. blocks & $D$ & II classes & Resolutions & Contractions \\
\hline \hline $4,3,2 ; Z_{2}$ & $A$ & 48 & $1^{4}$ & & & & \\
$4,3,4 ; Z_{2}$ & $B_{1}$ & 48 & $2^{4}$ & $d$ & & & \\
& $B_{2}$ & 24 & $2^{1} 1^{6}$ & $d$ & & & \\
& $B_{3}$ & 32 & $1^{8}$ & $d$ & & & \\
\hline $4,3,6 ; Z_{2}$ & $C_{1}$ & 48 & $3^{4}$ & $d$ & & & \\
& $C_{2}$ & 12 & $3^{1} 2^{3} 1^{3}$ & $d$ & & & \\
& $C_{3}$ & 16 & $2^{4} 1^{4}$ & $d$ & & & \\
& $C_{4}$ & 8 & $2^{2} 1^{8}$ & $d$ & & & \\
& $C_{5}$ & 48 & $1^{12}$ & $d$ & & & \\
\hline $4,3,6 ; Z_{3}$ & $D_{1}$ & 72 & $2^{4} 1^{4}$ & & 15 & 3 & \\
& $D_{2}$ & 18 & $2^{1} 1^{10}$ & & 6 & 0 & \\
& $D_{3}$ & 72 & $1^{12}$ & & 0 & 0 & \\
& $D_{4}$ & 144 & $1^{12}$ & & 48 & 204 & \\
& $D_{5}$ & 24 & $1^{12}$ & & 12 & 0 & \\
& $D_{6}$ & 12 & $1^{12}$ & & 6 & 0 & \\
\hline
\end{tabular}




\begin{tabular}{|c|c|c|c|c|c|c|c|}
\hline \multicolumn{8}{|c|}{ Numbers and properties of generated Bhaskar Rao designs } \\
\hline Parameters & Design & $|G|$ & Rep. blocks & $D$ & || classes & Resolutions & Contractions \\
\hline $4,3,4 ; Z_{4}$ & $E$ & 32 & $1^{8}$ & & & & $B_{3}$ \\
\hline $4,3,4 ; Z_{2}^{2}$ & $F$ & 96 & $1^{8}$ & & & & $B_{3}$ \\
\hline 4,$36 ; Z_{6}$ & $\begin{array}{l}G_{1} \\
G_{2} \\
G_{3} \\
G_{4} \\
G_{5} \\
G_{6} \\
G_{7} \\
G_{8} \\
G_{9} \\
G_{10} \\
G_{11} \\
G_{12} \\
G_{13} \\
G_{14} \\
G_{15} \\
G_{16} \\
G_{17} \\
G_{18} \\
G_{19} \\
G_{20} \\
G_{21} \\
G_{22}\end{array}$ & $\begin{array}{r}36 \\
144 \\
24 \\
36 \\
144 \\
48 \\
24 \\
48 \\
96 \\
72 \\
12 \\
12 \\
48 \\
12 \\
24 \\
48 \\
6 \\
6 \\
24 \\
6 \\
12 \\
24\end{array}$ & $\begin{array}{l}1^{12} \\
1^{12} \\
1^{12} \\
1^{12} \\
1^{12} \\
1^{12} \\
1^{12} \\
1^{12} \\
1^{12} \\
1^{12} \\
1^{12} \\
1^{12} \\
1^{12} \\
1^{12} \\
1^{12} \\
1^{12} \\
1^{12} \\
1^{12} \\
1^{12} \\
1^{12} \\
1^{12} \\
1^{12}\end{array}$ & & $\begin{array}{r}0 \\
0 \\
0 \\
0 \\
0 \\
96 \\
132 \\
216 \\
240 \\
420 \\
36 \\
48 \\
12 \\
12 \\
36 \\
12 \\
30 \\
30 \\
6 \\
54 \\
30 \\
30\end{array}$ & $\begin{array}{r}0 \\
0 \\
0 \\
0 \\
0 \\
270 \\
354 \\
?(\geqslant 1) \\
?(\geqslant 1) \\
?(\geqslant 1) \\
0 \\
9 \\
0 \\
0 \\
0 \\
0 \\
0 \\
0 \\
0 \\
0 \\
0 \\
0\end{array}$ & $\begin{array}{l}C_{5}, D_{3} \\
C_{5}, D_{3} \\
C_{4}, D_{3} \\
C_{2}, D_{3} \\
C_{1}, D_{3} \\
C_{5}, D_{4} \\
C_{4}, D_{4} \\
C_{3}, D_{4} \\
C_{3}, D_{4} \\
C_{2}, D_{4} \\
C_{5}, D_{5} \\
C_{5}, D_{5} \\
C_{5}, D_{5} \\
C_{4}, D_{5} \\
C_{4}, D_{5} \\
C_{3}, D_{5} \\
C_{5}, D_{6} \\
C_{5}, D_{6} \\
C_{5}, D_{6} \\
C_{4}, D_{6} \\
C_{4}, D_{6} \\
C_{3} D_{6}\end{array}$ \\
\hline $4,3,6 ; S_{3}$ & $\begin{array}{l}H_{1} \\
H_{2} \\
H_{3} \\
H_{4} \\
H_{5} \\
H_{6} \\
H_{7} \\
H_{8} \\
\end{array}$ & $\begin{array}{r}2592 \\
324 \\
72 \\
36 \\
96 \\
12 \\
18 \\
6\end{array}$ & $\begin{array}{l}1^{12} \\
1^{12} \\
1^{12} \\
1^{12} \\
1^{12} \\
1^{12} \\
1^{12} \\
1^{12}\end{array}$ & & $\begin{array}{r}864 \\
756 \\
420 \\
216 \\
240 \\
144 \\
54 \\
48\end{array}$ & $\begin{array}{r}?(\geqslant 1) \\
?(\geqslant 1) \\
?(\geqslant 1) \\
?(\geqslant 1) \\
?(\geqslant 1) \\
0 \\
0 \\
0\end{array}$ & $\begin{array}{l}C_{1} \\
C_{1} \\
C_{2} \\
C_{2} \\
C_{3} \\
C_{4} \\
C_{5} \\
C_{5}\end{array}$ \\
\hline $5,4,3 ; Z_{3}$ & $A$ & 360 & $1^{5}$ & & & & \\
\hline $5,4,6, Z_{3}$ & $\begin{array}{l}B_{1} \\
B_{2} \\
B_{3} \\
B_{4} \\
B_{5} \\
B_{6} \\
B_{7} \\
B_{8} \\
B_{9}\end{array}$ & $\begin{array}{r}360 \\
120 \\
72 \\
48 \\
24 \\
36 \\
36 \\
12 \\
24\end{array}$ & $\begin{array}{l}2^{5} \\
1^{10} \\
2^{1} 1^{8} \\
2^{1} 1^{8} \\
1^{10} \\
1^{10} \\
1^{10} \\
1^{10} \\
2^{1} 1^{8}\end{array}$ & $\begin{array}{l}d \\
d \\
d \\
d \\
d \\
d \\
d \\
d\end{array}$ & & & \\
\hline $5,4,6 ; Z_{2}$ & C & 10 & $1^{10}$ & & & & \\
\hline $5,4,6 ; S_{3}$ & $D$ & 30 & $1^{10}$ & & & & $C$ \\
\hline $5,4,6 ; Z_{6}$ & - & & & & & & \\
\hline $6,5,4 ; Z_{2}$ & $A$ & 240 & $1^{6}$ & & & & \\
\hline
\end{tabular}


We now investigate various structures derived from some of the generated designs.

A generalized Room square $G R S(r, \lambda ; \nu)$ is an $r \times r$ array on a finite $\nu$-set $V$ of elements such that: (i) every cell of the array contains a subset (possibly empty) of $V$; (ii) every element of $V$ is contained in precisely one cell of each row and column; and (iii) every pair of distinct elements of $V$ is contained in exactly $\lambda$ cells of the array. A GRS is said to be uniform if all subsets have the same cardinality. A GRS is equivalent to a doubly resolvable $(r, \lambda)$-design. To see this, suppose we have two orthogonal resolutions $R=\left\{R_{1}, \ldots, R_{u}\right\}, R^{\prime}=$ $\left\{R_{1}^{\prime}, \ldots, R_{u}^{\prime}\right\}$ of the same $(r, \lambda)$-design $D$. Then we can form a $G R S(u, \lambda ; \nu) S$ in which block $B$ of $D$ is placed in cell $(i, j)$ of $S$ if it belongs to parallel classes $R_{i}$ and $R_{j}^{\prime}$. An equidistant permutation array $\operatorname{EP} A(r, \lambda ; \nu)$ is a $\nu \times r$ array defined on an $r$-set of elements such that: (i) every row of the array is a permutation of the elements of $V$; and (ii) every pair of distinct rows of the array have precisely $\lambda$ common column entries. An $E P A(r, \lambda ; v)$ is equivalent to a $G R S(r, \lambda ; \nu)$. To see this, let $V=\{1,2, \ldots, \nu\}$ be the element set of both a $G R S S$ and the corresponding EPA $A$. Then the $(i, j)$ th entry of $A$ is $k \in V$ if and only if the element $i$ appears in the $(k, j)$ th cell of $S$ (see [19]).

From a $G D D(\nu \times g, k, \lambda)$ we can construct an $(r, \lambda)$-design $D$ on the same element set $X|X|=\nu g$ by adjoining $\lambda$ copies of the groups to the blocks of the $G D D$. We note that $D$ has $r=\lambda+\lambda g(\nu-1) /(k-1)$, and two block sizes $k$ and $g$. If $D$ happens to be doubly resolvable then it can be used to construct a $G R S(r, \lambda ; \nu g)$.

The following two examples illustrate such a derivation process using appropriate GBRD's.

Firstly, consider the $\operatorname{GBRD}\left(4,3,6 ; Z_{3}\right) D_{4}$ from the appendices. A computer analysis reveals that the corresponding $G D D(4 \times 3,3,2)$ is resolvable. Since the groups and blocks both have size 3 the $(11,2)$-design derivable from the $G D D$ is in fact a $B I B D(12,44,11,3,2)$, also called a twofold triple system ([4]). This system is doubly resolvable; one resolution is inherited from the original GDD with two additional parallel classes formed by the groups, while the other resolution is derived from the block orbits of the GDD under $Z_{3}$. The obtained system solves the existence problem for doubly resolvable twofold triple systems with $\nu=12$, the smallest previously unknown order [4]. The system is important since it forms a basis for recursive constructions of twofold triple systems. We present it here as a uniform $\operatorname{GRS}(11,2 ; 12)$ : 


\begin{tabular}{|c|c|c|c|c|c|c|c|c|c|c|c|}
\hline & 1 & 2 & 3 & 4 & 5 & 6 & 7 & 8 & 9 & 10 & 11 \\
\hline 1 & $A B C$ & & $D E F$ & & $\widehat{G H I}$ & & $J K L$ & & & & \\
\hline 2 & & $A B C$ & & $D E F$ & & $G H I$ & & $J K L$ & & & \\
\hline 3 & $F G J$ & & & $A I L$ & $B E K$ & & $C D H$ & & & & \\
\hline 4 & $E I L$ & & & $C H K$ & & $B F J$ & & & $A D G$ & & \\
\hline 5 & $D H K$ & & & & & $A E L$ & $B F G$ & & & $C I J$ & \\
\hline 6 & & $F H L$ & & $B G J$ & & $C D K$ & $A E I$ & & & & \\
\hline 7 & & $E G K$ & & & $A D J$ & & & & $B H L$ & & $C F I$ \\
\hline 8 & & $D I J$ & & & $C F L$ & & & & & $B E H$ & $A G K$ \\
\hline 9 & & & $B I K$ & & & & & $A F H$ & $C E J$ & $D G L$ & \\
\hline 10 & & & $A H J$ & & & & & $C E G$ & $F I K$ & & $B D L$ \\
\hline 11 & & & $C G L$ & & & & & $B D I$ & & $A F K$ & $E H J$ \\
\hline
\end{tabular}

Secondly, consider the $\operatorname{GBR} D\left(4,3,6 ; Z_{6}\right) G_{10}$ from the appendices. Adding the groups to the blocks of the corresponding $G D D(4 \times 6,3,1)$ yields a $(10,1)$-design with two block sizes, 3 and 6 . This design turns out to be doubly resolvable and can be presented as a $\operatorname{GRS}(10,1 ; 24)$ :

\begin{tabular}{|c|c|c|c|c|c|c|c|c|c|c|}
\hline & 1 & 2 & 3 & 4 & 5 & 6 & 7 & 8 & 9 & 10 \\
\hline 1 & $A M S$ & $E K X$ & & $J R U$ & & $D G Q$ & LOV & $F P T$ & $B I W$ & $C H N$ \\
\hline 2 & $E H R$ & $B N T$ & $F L S$ & & $K M V$ & & $G P W$ & $A Q U$ & $C J X$ & DIO \\
\hline 3 & & $F I M$ & $\mathrm{COU}$ & $A G T$ & & $L N W$ & $H Q X$ & $B R V$ & $D K S$ & $E J P$ \\
\hline 4 & $G O X$ & & $A J N$ & $D P V$ & $B H U$ & & $I R S$ & $C M W$ & $E L T$ & $F K Q$ \\
\hline 5 & & $H P S$ & & $B K O$ & $E Q W$ & $C I V$ & $J M T$ & $D N X$ & $F G U$ & $A L R$ \\
\hline 6 & $D J W$ & & $I Q T$ & & $C L P$ & $F R X$ & $K N U$ & EOS & $A H V$ & $B G M$ \\
\hline 7 & $I P U$ & $J Q V$ & $K R W$ & $L M \bar{X}$ & $\overline{G N S}$ & HOT & $\overline{A B C D E F}$ & & & \\
\hline 8 & $F N V$ & $A O W$ & $B P X$ & $C Q S$ & $D R T$ & $E M U$ & & GHIJKL & & \\
\hline 9 & $C K T$ & $D L U$ & $E G V$ & $F H W$ & $A I X$ & $B J S$ & & & $M N O P Q R$ & \\
\hline 10 & $B L Q$ & $C G R$ & $D H M$ & $E I N$ & FJO & $A K P$ & & & & STUVWX \\
\hline
\end{tabular}


From the above GRS we can form an $\operatorname{EPA}(10,1 ; 24)$ to which three more permutations can be added yielding an $E P A(10,1 ; 27)$ :

\begin{tabular}{|llllllllll|}
\hline 1 & 2 & 3 & 4 & 5 & 6 & 7 & 8 & 9 & 0 \\
5 & 6 & 1 & 2 & 3 & 4 & 8 & 0 & 9 & 7 \\
6 & 1 & 2 & 3 & 4 & 5 & 7 & 0 & 8 & 9 \\
3 & 6 & 4 & 8 & 0 & 9 & 7 & 1 & 2 & 5 \\
9 & 4 & 1 & 5 & 8 & 0 & 7 & 2 & 3 & 6 \\
0 & 9 & 5 & 2 & 6 & 8 & 7 & 3 & 4 & 1 \\
8 & 0 & 9 & 6 & 3 & 1 & 7 & 4 & 5 & 2 \\
2 & 8 & 0 & 9 & 1 & 4 & 7 & 5 & 6 & 3 \\
5 & 3 & 8 & 0 & 9 & 2 & 7 & 6 & 1 & 4 \\
0 & 4 & 8 & 7 & 3 & 6 & 1 & 5 & 2 & 9 \\
1 & 0 & 5 & 8 & 7 & 4 & 2 & 6 & 3 & 9 \\
5 & 2 & 0 & 6 & 8 & 7 & 3 & 1 & 4 & 9 \\
7 & 6 & 3 & 0 & 1 & 8 & 4 & 2 & 5 & 9 \\
8 & 7 & 1 & 4 & 0 & 2 & 5 & 3 & 6 & 9 \\
3 & 8 & 7 & 2 & 5 & 0 & 6 & 4 & 1 & 9 \\
8 & 4 & 3 & 9 & 6 & 7 & 2 & 0 & 1 & 5 \\
7 & 8 & 5 & 4 & 9 & 1 & 3 & 0 & 2 & 6 \\
2 & 7 & 8 & 6 & 5 & 9 & 4 & 0 & 3 & 1 \\
9 & 3 & 7 & 8 & 1 & 6 & 5 & 0 & 4 & 2 \\
1 & 9 & 4 & 7 & 8 & 2 & 6 & 0 & 5 & 3 \\
3 & 2 & 9 & 5 & 7 & 8 & 1 & 0 & 6 & 4 \\
7 & 2 & 4 & 9 & 3 & 0 & 5 & 6 & 8 & 1 \\
0 & 7 & 3 & 5 & 9 & 4 & 6 & 1 & 8 & 2 \\
5 & 0 & 7 & 4 & 6 & 9 & 1 & 2 & 8 & 3 \\
9 & 6 & 0 & 7 & 5 & 1 & 2 & 3 & 8 & 4 \\
2 & 9 & 1 & 0 & 7 & 6 & 3 & 4 & 8 & 5 \\
1 & 3 & 9 & 2 & 0 & 7 & 4 & 5 & 8 & 6 \\
\hline
\end{tabular}

This greatly improves the previously known maximum value of $\nu$, viz $\nu=16$, for which an $\operatorname{EPA}(10,1 ; \nu)$ exists [19].

\section{Acknowledgements}

We would like to thank Wendy Myrvold for pointing out the existence of a 27th permutation in the $\operatorname{EPA}(10,1 ; 27)$. We would also like to thank Norman $\mathrm{L}$. Johnson for supplying information used in Construction 2 concerning projective planes which admit a collineation group fixing a Baer subplane pointwise. 


\section{Appendices}

\section{A1. Group multiplication tables}

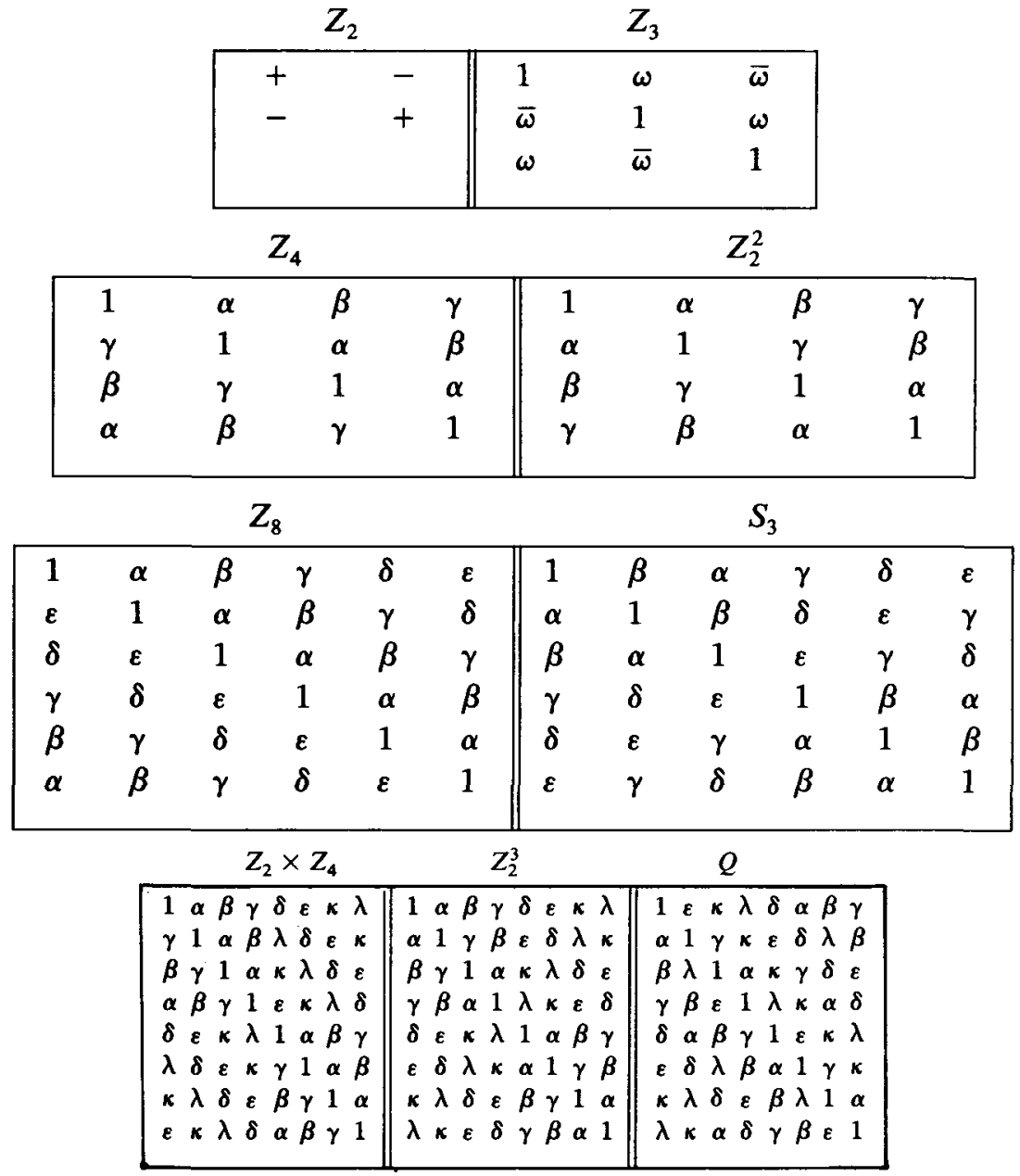


A2. Enumerated $G B R D(k+1, k, l(k-1) ; G)$ 's

1. $\operatorname{GBRD}\left(4,3,2 ; Z_{2}\right)$

\begin{tabular}{lllll}
1 & 1 & 1 & 1 \\
1 & + & + & - & 0 \\
1 & + & - & 0 & + \\
1 & + & 0 & + & - \\
0 & + & + & + \\
\hline
\end{tabular}

2. $G B R D\left(4,3,4 ; Z_{2}\right)$ 's

\begin{tabular}{|c|c|c|c|c|c|c|c|c|c|c|c|c|c|c|c|c|c|}
\hline$B_{1}$ & 1 & 1 & 1 & 1 & 1 & 1 & 1 & 1 & $B_{2}$ & 1 & 2 & 2 & 2 & 1 & 2 & 2 & 2 \\
\hline 1 & + & + & - & 0 & + & + & - & 0 & 1 & + & + & - & 0 & + & - & + & 0 \\
\hline 1 & + & - & 0 & + & + & - & 0 & + & 1 & + & - & 0 & + & + & + & 0 & - \\
\hline 1 & + & 0 & + & - & + & 0 & + & - & 1 & + & 0 & + & - & + & 0 & - & + \\
\hline 1 & 0 & + & + & + & 0 & + & + & + & 2 & 0 & + & + & + & 0 & + & + & + \\
\hline & & $B_{3}$ & & 1 & 1 & & 1 & 1 & & 1 & 1 & & 1 & 1 & & & \\
\hline & & 1 & & + & + & & - & 0 & & + & + & & + & 0 & & & \\
\hline & & 1 & & + & - & & 0 & + & & + & - & & 0 & - & & & \\
\hline & & 1 & & + & 0 & & + & - & & - & 0 & & + & - & & & \\
\hline & & 1 & & 0 & + & & + & + & & 0 & - & & + & + & & & \\
\hline
\end{tabular}

3. $G B R D\left(4,3,6 ; Z_{2}\right)$ 's

\begin{tabular}{|c|c|c|c|c|c|c|c|c|c|c|c|c|c|c|c|c|c|c|c|c|c|c|c|c|c|}
\hline$C_{1}$ & & 1 & 1 & 1 & 1 & 1 & 1 & 1 & 1 & 1 & 1 & 1 & $C_{2}$ & 1 & 2 & 2 & & 1 & 2 & 2 & 2 & 1 & 3 & 3 & 3 \\
\hline 1 & + & + & - & 0 & + & + & - & 0 & + & + & - & 0 & 1 & + & + & - & 0 & + & + & - & 0 & + & - & + & 0 \\
\hline 1 & + & - & 0 & + & + & - & 0 & + & + & - & 0 & + & 1 & + & - & 0 & + & + & 0 & 0 & + & + & + & 0 & - \\
\hline 1 & + & 0 & + & - & + & 0 & + & - & + & 0 & + & - & 1 & + & 0 & + & - & + & 0 & + & - & + & 0 & - & + \\
\hline 1 & 0 & + & + & + & 0 & + & + & + & 0 & + & + & + & 2 & 0 & + & + & + & 0 & + & + & + & 0 & + & + & + \\
\hline
\end{tabular}

$\begin{array}{llllllllllllllllllllllllll}C_{3} & 1 & 1 & 1 & 1 & 1 & 1 & 1 & 1 & 2 & 2 & 2 & 2 & C_{4} & 1 & 1 & 2 & 2 & 1 & 3 & 4 & 4 & 3 & 1 & 4 & 4\end{array}$

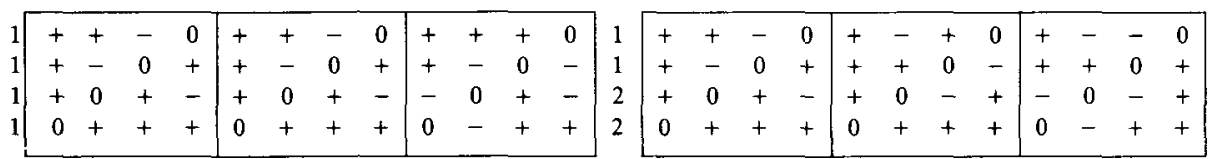

\begin{tabular}{l|cccc|cccc|cccc|}
$C_{5}$ & 1 & 1 & 1 & 1 & 1 & 1 & 1 & 1 & 1 & 1 & 1 & 1 \\
1 & + & + & - & 0 & + & + & + & 0 & + & - & - & 0 \\
1 & + & - & 0 & + & + & - & 0 & - & - & - & 0 & + \\
1 & + & 0 & + & - & - & 0 & + & - & + & 0 & + & + \\
1 & 0 & + & + & + & 0 & - & + & + & 0 & + & - & + \\
\cline { 2 - 7 }
\end{tabular}


4. $G B R D\left(4,3,6 ; Z_{3}\right)$ 's

$\begin{array}{llllllllllllllllllllllllll}D_{1} & 1 & 2 & 2 & 1 & 2 & 2 & 1 & 2 & 2 & 1 & 2 & 2 & D_{2} & 1 & 1 & 2 & 3 & 4 & 4 & 3 & 4 & 4 & 3 & 4 & 4\end{array}$ \begin{tabular}{lllllllllllll|l|llllllllllll|}
1 & 1 & $\omega$ & $\omega$ & 1 & 1 & 1 & $\bar{\omega}$ & 1 & 1 & 0 & 0 & 0 & 1 & 1 & 1 & 1 & 1 & $\omega$ & 1 & $\omega$ & 1 & $\bar{\omega}$ & 0 & 0 & 0 \\
1 & 1 & 1 & 1 & $\omega$ & $\omega$ & 0 & 0 & 0 & $\bar{\omega}$ & 1 & 1 & 1 & 1 & $\omega$ & $\omega$ & 1 & $\bar{\omega}$ & 0 & 0 & 0 & 1 & $\omega$ & 1 \\
1 & $\omega$ & 1 & 1 & 0 & 0 & 0 & 1 & 1 & 1 & 1 & $\bar{\omega}$ & $\bar{\omega}$ & 1 & 1 & $\bar{\omega}$ & 0 & 0 & 0 & 1 & $\omega$ & 1 & $\omega$ & 1 & $\bar{\omega}$ \\
1 & 0 & 0 & $\omega$ & 1 & 1 & 1 & $\bar{\omega}$ & $\bar{\omega}$ & 1 & 1 & 1 & 2 & 0 & 0 & $\bar{\omega}$ & 1 & 1 & $\bar{\omega}$ & 1 & 1 & $\bar{\omega}$ & 1 & 1 \\
\hline
\end{tabular} $\begin{array}{lllllllllllllllllllllllllll}D_{3} & 1 & 1 & 1 & 1 & 1 & 1 & 1 & 1 & 1 & 1 & 1 & 1 & D_{4} & 1 & 2 & 2 & 1 & 2 & 2 & 1 & 2 & 2 & 1 & 2 & 2\end{array}$

\begin{tabular}{lllllllllllll|l|llllllllllll|}
1 \\
1 \\
1 & 1 & 1 & $\omega$ & 1 & 1 & $\omega$ & $\omega$ & 1 & 1 & 0 & 0 & 0 \\
1 & $\omega$ & 1 & 1 & $\omega$ & 1 & 0 & 0 & 0 & $\omega$ & 1 & 1 & 1 & 1 & 1 & $\omega$ & 1 & 1 & $\omega$ & $\bar{\omega}$ & 1 & 1 & 0 & 0 & 0 \\
1 & 1 & 1 & 0 & 0 & 0 & 1 & 1 & $\omega$ & 1 & 1 & $\omega$ & $\omega$ & 1 & 1 & $\omega$ & 1 & 0 & 0 & 0 & $\bar{\omega}$ & 1 & 1 \\
1 & 0 & 0 & $\omega$ & 1 & 1 & 1 & $\omega$ & 1 & 1 & $\omega$ & 1 & 1 & 1 & 0 & 0 & 0 & 1 & 1 & $\bar{\omega}$ & 1 & 1 & $\bar{\omega}$ \\
0 & 0 & 0 & 0 & 0 & $\omega$ & 1 & 1 & 1 & $\bar{\omega}$ & 1 & 1 & $\bar{\omega}$ & 1 \\
\hline
\end{tabular}
$\begin{array}{llllllllllllllllllllllllll}D_{5} & 1 & 2 & 2 & 1 & 2 & 2 & 1 & 2 & 2 & 1 & 2 & 2 & D_{6} & 1 & 2 & 3 & 1 & 2 & 3 & 1 & 2 & 3 & 1 & 2 & 3\end{array}$ \begin{tabular}{l|llllllllllll|l|lllllllllllll|}
1 & $\omega$ & 1 & 1 & 1 & 1 & $\bar{\omega}$ & 1 & 1 & $\bar{\omega}$ & 0 & 0 & 0 \\
1 & 1 & 1 & $\bar{\omega}$ & $\omega$ & 1 & 1 & 0 & 0 & 0 & 1 & 1 & $\bar{\omega}$ & 1 & 1 & $\bar{\omega}$ & 1 & 1 & 1 & 1 & $\omega$ & 1 & $\bar{\omega}$ & 0 & 0 & 0 \\
$\omega$ & 1 & $\bar{\omega}$ & 1 & $\bar{\omega}$ & 1 & 0 & 0 & 0 & 1 & 1 & 1 \\
1 & $\bar{\omega}$ & 1 & 0 & 0 & 0 & $\omega$ & 1 & 1 & 1 & $\bar{\omega}$ & 1 & 1 & 1 & 1 & 1 & 0 & 0 & 0 & 1 & $\bar{\omega}$ & 1 & $\omega$ & 1 & $\bar{\omega}$ \\
0 & 0 & 0 & 1 & $\bar{\omega}$ & 1 & 1 & $\bar{\omega}$ & 1 & $\omega$ & 1 & 1 & 1 & 0 & 0 & 0 & $\omega$ & 1 & $\bar{\omega}$ & 1 & 1 & 1 & 1 & $\bar{\omega}$ & 1 \\
\hline
\end{tabular}

\section{5. $G B R D\left(4,3,4 ; Z_{4}\right) \quad$ 6. $G B R D\left(4,3,4 ; Z_{2}^{2}\right)$}

$\begin{array}{llllllllllllllllll}E & 1 & 2 & 1 & 2 & 1 & 2 & 1 & 2 & F & 1 & 1 & 1 & 1 & 1 & 1 & 1 & 1\end{array}$

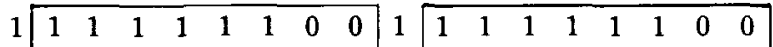

$1 \quad \begin{array}{llllllllllllllllll}1 & \alpha & \beta & \gamma & 0 & 0 & 1 & 1 & 1 & 1 & \alpha & \beta & \gamma & 0 & 0 & 1 & 1\end{array}$

$1 \quad$\begin{tabular}{llllllll|l|llllllll}
1 & $\beta$ & 0 & 0 & $\gamma$ & $\alpha$ & $\beta$ & $\gamma$ & 1 & 1 & $\beta$ & 0 & 0 & $\gamma$ & $\alpha$ & $\alpha$ & $\beta$
\end{tabular}

$1 \quad$\begin{tabular}{llllllll|lllllllll}
0 & 0 & 1 & $\gamma$ & $\beta$ & $\alpha$ & $\gamma$ & $\alpha$ & 1 & 0 & 0 & $\gamma$ & 1 & $\beta$ & $\alpha$ & $\beta$ & 1
\end{tabular}

7. $G B R D\left(4,3,6 ; Z_{6}\right)$ 's

$\begin{array}{llllllllllllllllllllllllll}G_{1} & 1 & 2 & 2 & 1 & 2 & 2 & 1 & 2 & 2 & 3 & 3 & 3 & G_{2} & 1 & 1 & 1 & 1 & 1 & 1 & 1 & 1 & 1 & 1 & 1 & 1\end{array}$

\begin{tabular}{|c|c|c|c|c|c|c|c|c|c|c|c|c|c|c|c|c|c|c|c|c|c|c|c|c|}
\hline & 1 & 1 & 1 & 1 & 1 & 1 & 1 & 1 & 0 & 0 & 0 & 1 & 1 & 1 & 1 & 1 & 1 & 1 & 1 & 1 & 1 & 0 & 0 & 0 \\
\hline & $\alpha$ & $\beta$ & $\gamma$ & $\delta$ & $\varepsilon$ & 0 & 0 & 0 & 1 & 1 & 1 & 1 & 1 & $\alpha$ & $\beta$ & $\gamma$ & $\delta$ & $\varepsilon$ & 0 & 0 & 0 & 1 & 1 & 1 \\
\hline & $\beta$ & $\alpha$ & 0 & 0 & 0 & $\gamma$ & $\delta$ & $\varepsilon$ & $\boldsymbol{\beta}$ & $\gamma$ & $\delta$ & 1 & 1 & $\beta$ & $\alpha$ & 0 & 0 & 0 & $\gamma$ & $\delta$ & $\varepsilon$ & $\beta$ & $\gamma$ & $\delta$ \\
\hline & 0 & 0 & 1 & $\boldsymbol{\beta}$ & $\alpha$ & $\gamma$ & $\varepsilon$ & $\delta$ & 1 & $\varepsilon$ & $\alpha$ & 1 & 0 & 0 & 0 & 1 & $\beta$ & $\alpha$ & $\gamma$ & $\boldsymbol{\varepsilon}$ & $\delta$ & $\varepsilon$ & $\alpha$ & 1 \\
\hline
\end{tabular}

$\begin{array}{llllllllllllllllllllllllll}G_{3} & 1 & 2 & 1 & 1 & 2 & 1 & 3 & 4 & 3 & 3 & 4 & 3 & G_{4} & 1 & 2 & 1 & 1 & 2 & 1 & 3 & 3 & 3 & 1 & 2 & 1\end{array}$

\begin{tabular}{lllllllllllll|l|llllllllllll|}
1 & 1 & 1 & 1 & 1 & 1 & 1 & 1 & 1 & 1 & 0 & 0 & 0 \\
1 & 1 & $\alpha$ & $\beta$ & $\gamma$ & $\delta$ & $\varepsilon$ & 0 & 0 & 0 & 1 & 1 & 1 & 1 \\
2 & 1 & $\beta$ & $\alpha$ & 0 & 0 & 0 & $\gamma$ & $\delta$ & $\varepsilon$ & $\beta$ & $\gamma$ & $\delta$ \\
2 & 0 & 0 & 1 & $\beta$ & $\alpha$ & $\varepsilon$ & $\delta$ & $\gamma$ & $\alpha$ & 1 & $\varepsilon$ & 1 & 1 & 1 & 1 & 1 & 1 & 1 & 1 & 0 & 0 & 0 \\
1 & $\alpha$ & $\beta$ & $\gamma$ & $\delta$ & $\varepsilon$ & 0 & 0 & 0 & 1 & 1 & 1 \\
1 & $\beta$ & $\delta$ & 0 & 0 & 0 & $\alpha$ & $\gamma$ & $\varepsilon$ & $\gamma$ & $\delta$ & $\varepsilon$ \\
0 & 0 & 0 & 1 & $\beta$ & $\delta$ & $\alpha$ & $\varepsilon$ & $\gamma$ & $\beta$ & $\alpha$ & 1 \\
\hline
\end{tabular}


$\begin{array}{llllllllllllllllllllllllll}G_{5} & 1 & 1 & 1 & 1 & 1 & 1 & 1 & 1 & 1 & 1 & 1 & 1 & G_{6} & 1 & 2 & 1 & 1 & 2 & 1 & 1 & 2 & 1 & 1 & 2 & 1\end{array}$ \begin{tabular}{lllllllllllll|l|lllllllllllll}
1 & 1 & 1 & 1 & 1 & 1 & 1 & 1 & 1 & 1 & 0 & 0 & 0 & 1 & 1 & 1 & 1 & 1 & 1 & 1 & 1 & 1 & 1 & 0 & 0 & 0
\end{tabular} \begin{tabular}{lllllllllllll|l|lllllllllllll}
1 & 1 & $\beta$ & $\delta$ & $\alpha$ & $\gamma$ & $\varepsilon$ & 0 & 0 & 0 & 1 & 1 & 1 & 1 & 1 & $\alpha$ & $\beta$ & $\gamma$ & $\delta$ & $\varepsilon$ & 0 & 0 & 0 & 1 & 1 & 1
\end{tabular} \begin{tabular}{lllllllllllll|l|llllllllllll}
1 & 1 & $\delta$ & $\beta$ & 0 & 0 & 0 & $\alpha$ & $\gamma$ & $\varepsilon$ & $\alpha$ & $\gamma$ & $\varepsilon$ & 1 & 1 & $\beta$ & $\alpha$ & 0 & 0 & 0 & $\gamma$ & $\varepsilon$ & $\delta$ & $\beta$ & $\delta$ & $\gamma$
\end{tabular} \begin{tabular}{lllllllllllll|l|llllllllllll}
1 & 0 & 0 & 0 & 1 & $\delta$ & $\beta$ & $\alpha$ & $\varepsilon$ & $\gamma$ & 1 & $\delta$ & $\beta$ & 1 & 0 & 0 & 0 & 1 & $\beta$ & $\alpha$ & $\delta$ & $\varepsilon$ & $\gamma$ & 1 & $\alpha$ & $\varepsilon$
\end{tabular}

$\begin{array}{llllllllllllllllllllllllll}G_{7} & 1 & 2 & 2 & 3 & 4 & 5 & 3 & 4 & 5 & 1 & 2 & 2 & G_{8} & 1 & 2 & 3 & 1 & 2 & 3 & 1 & 2 & 3 & 1 & 2 & 3\end{array}$

\begin{tabular}{lllllllllllll|l|llllllllllll|}
1 & 1 & 1 & 1 & 1 & 1 & 1 & 1 & 1 & 1 & 0 & 0 & 0 \\
2 & 1 & $\alpha$ & $\beta$ & $\gamma$ & $\delta$ & $\varepsilon$ & 0 & 0 & 0 & 1 & 1 & 1 & 1 & 1 & 1 & 1 & 1 & 1 & 1 & 1 & 1 & 1 & 0 & 0 & 0 \\
1 & 1 & $\beta$ & $\alpha$ & 0 & 0 & 0 & $\gamma$ & $\delta$ & $\varepsilon$ & $\gamma$ & $\beta$ & $\delta$ & $\beta$ & $\gamma$ & $\delta$ & $\varepsilon$ & 0 & 0 & 0 & 1 & 1 & 1 \\
1 & 0 & 0 & 0 & 1 & $\beta$ & $\delta$ & $\gamma$ & $\varepsilon$ & $\alpha$ & 1 & $\alpha$ & $\beta$ & 1 & $\beta$ & $\delta$ & 0 & 0 & 0 & $\varepsilon$ & $\gamma$ & $\alpha$ & $\gamma$ & $\delta$ & $\varepsilon$ \\
1 & 0 & 0 & 1 & $\varepsilon$ & $\delta$ & $\gamma$ & $\beta$ & $\alpha$ & $\delta$ & 1 & $\beta$ \\
\hline
\end{tabular}

$\begin{array}{llllllllllllllllllllllllll}G_{9} & 1 & 2 & 1 & 1 & 2 & 1 & 1 & 2 & 1 & 1 & 2 & 1 & G_{10} & 1 & 2 & 1 & 1 & 2 & 1 & 3 & 4 & 3 & 1 & 2 & 1\end{array}$

$1 \begin{array}{lllllllllllllllllllllllllll}1 & 1 & 1 & 1 & 1 & 1 & 1 & 1 & 1 & 0 & 0 & 0 & 1 & 1 & 1 & 1 & 1 & 1 & 1 & 1 & 1 & 1 & 0 & 0 & 0\end{array}$ \begin{tabular}{lllllllllllll|l|llllllllllll}
1 & 1 & $\alpha$ & $\beta$ & $\gamma$ & $\delta$ & $\varepsilon$ & 0 & 0 & 0 & 1 & 1 & 1 & 1 & 1 & $\alpha$ & $\beta$ & $\gamma$ & $\delta$ & $\varepsilon$ & 0 & 0 & 0 & 1 & 1 & 1
\end{tabular}

\begin{tabular}{lllllllllllll|l|llllllllllll}
1 & 1 & $\beta$ & $\delta$ & 0 & 0 & 0 & $\alpha$ & $\varepsilon$ & $\gamma$ & $\gamma$ & $\delta$ & $\varepsilon$ & 2 & 1 & $\beta$ & $\delta$ & 0 & 0 & 0 & $\alpha$ & $\varepsilon$ & $\gamma$ & $\gamma$ & $\delta$ & $\varepsilon$
\end{tabular} $1\left[\begin{array}{llllllllllll}0 & 0 & 0 & 1 & \varepsilon & \delta & \gamma & \beta & \alpha & \beta & \delta & 1\end{array}\right] \begin{array}{lllllllllllll}0 & 0 & 0 & 1 & \beta & \delta & \gamma & \varepsilon & \alpha & \beta & \alpha & 1\end{array}$ $\begin{array}{llllllllllllllllllllllllll}G_{11} & 1 & 2 & 3 & 1 & 2 & 3 & 4 & 5 & 5 & 6 & 7 & 7 & G_{12} & 1 & 2 & 3 & 4 & 5 & 6 & 1 & 2 & 3 & 4 & 5 & 6\end{array}$ \begin{tabular}{lllllllllllll|l|llllllllllll|}
1 & 1 & 1 & 1 & 1 & 1 & 1 & 1 & 1 & 1 & 0 & 0 & 0 \\
2 & 1 & $\alpha$ & $\beta$ & $\gamma$ & $\delta$ & $\varepsilon$ & 0 & 0 & 0 & 1 & 1 & 1 \\
3 & 1 & $\beta$ & $\varepsilon$ & 0 & 0 & 0 & $\alpha$ & $\gamma$ & $\delta$ & $\beta$ & $\delta$ & $\varepsilon$ & 1 & 1 & 1 & 1 & 1 & 1 & 1 & 1 & 1 & 0 & 0 & 0 \\
1 & 1 & $\alpha$ & $\beta$ & $\gamma$ & $\delta$ & $\varepsilon$ & 0 & 0 & 0 & 1 & 1 & 1 \\
1 & $\beta$ & $\varepsilon$ & 0 & 0 & 0 & $\gamma$ & $\alpha$ & $\delta$ & $\delta$ & $\beta$ & $\varepsilon$ \\
0 & 0 & 0 & 1 & $\beta$ & $\varepsilon$ & $\alpha$ & $\delta$ & $\gamma$ & $\varepsilon$ & $\beta$ & $\alpha$ & 2 & 0 & 1 & $\beta$ & $\varepsilon$ & $\delta$ & $\gamma$ & $\alpha$ & $\beta$ & $\alpha$ & $\varepsilon$ \\
\hline
\end{tabular} $\begin{array}{lllllllllllllllllllllllllll}G_{13} & 1 & 2 & 1 & 1 & 2 & 1 & 1 & 2 & 1 & 1 & 2 & 1 & G_{14} & 1 & 2 & 3 & 1 & 2 & 3 & 4 & 5 & 5 & 6 & 7 & 7\end{array}$ \begin{tabular}{lllllllllllll|l|llllllllllll|}
1 & 1 & 1 & 1 & 1 & 1 & 1 & 1 & 1 & 1 & 0 & 0 & 0 \\
1 & 1 & $\alpha$ & $\beta$ & $\gamma$ & $\delta$ & $\varepsilon$ & 0 & 0 & 0 & 1 & 1 & 1 \\
1 & 1 & $\gamma$ & $\alpha$ & 0 & 0 & 0 & $\delta$ & $\beta$ & $\varepsilon$ & $\gamma$ & $\alpha$ & $\delta$ & 1 & 1 & 1 & 1 & 1 & 1 & 1 & 1 & 1 & 0 & 0 & 0 \\
1 & $\alpha$ & $\beta$ & $\gamma$ & $\delta$ & $\varepsilon$ & 0 & 0 & 0 & 1 & 1 & 1 \\
1 & 0 & 0 & 0 & 1 & $\gamma$ & $\alpha$ & $\varepsilon$ & $\beta$ & $\delta$ & $\alpha$ & $\delta$ & 1 & $\beta$ & $\varepsilon$ & 0 & 0 & 0 & $\delta$ & $\alpha$ & $\gamma$ & $\varepsilon$ & $\beta$ & $\delta$ \\
0 & 0 & 0 & 1 & $\beta$ & $\varepsilon$ & $\delta$ & $\gamma$ & $\alpha$ & $\beta$ & $\alpha$ & $\varepsilon$ \\
\hline
\end{tabular} $\begin{array}{llllllllllllllllllllllllll}G_{15} & 1 & 2 & 1 & 1 & 2 & 1 & 3 & 4 & 3 & 3 & 4 & 3 & G_{16} & 1 & 2 & 2 & 1 & 2 & 2 & 1 & 2 & 2 & 1 & 2 & 2\end{array}$ \begin{tabular}{lllllllllllll|l|lllllllllllll|}
1 & 1 & 1 & 1 & 1 & 1 & 1 & 1 & 1 & 1 & 0 & 0 & 0 \\
1 & 1 & $\alpha$ & $\beta$ & $\gamma$ & $\delta$ & $\varepsilon$ & 0 & 0 & 0 & 1 & 1 & 1 \\
2 & 1 & $\gamma$ & $\alpha$ & 0 & 0 & 0 & $\beta$ & $\varepsilon$ & $\delta$ & $\alpha$ & $\delta$ & $\gamma$ & 1 & 1 & 1 & 1 & 1 & 1 & 1 & 1 & 1 & 0 & 0 & 0 \\
1 & $\alpha$ & $\gamma$ & $\varepsilon$ & $\beta$ & $\delta$ & 0 & 0 & 0 & 1 & 1 & 1 \\
1 & $\gamma$ & $\gamma$ & $\alpha$ & 0 & 0 & 0 & $\varepsilon$ & $\beta$ & $\delta$ & $\gamma$ & $\alpha$ & $\varepsilon$ \\
0 & 0 & 1 & $\gamma$ & $\alpha$ & $\delta$ & $\varepsilon$ & $\beta$ & 1 & $\alpha$ & $\delta$ & 1 & 0 & $\beta$ & 1 & $\delta$ & $\varepsilon$ & $\gamma$ & $\alpha$ & $\beta$ & $\varepsilon$ & $\alpha$ \\
\hline
\end{tabular} $\begin{array}{llllllllllllllllllllllllll}G_{17} & 1 & 2 & 3 & 4 & 5 & 6 & 7 & 8 & 9 & 10 & 11 & 12 & G_{18} & 1 & 2 & 3 & 4 & 5 & 6 & 7 & 8 & 9 & 10 & 11 & 12\end{array}$

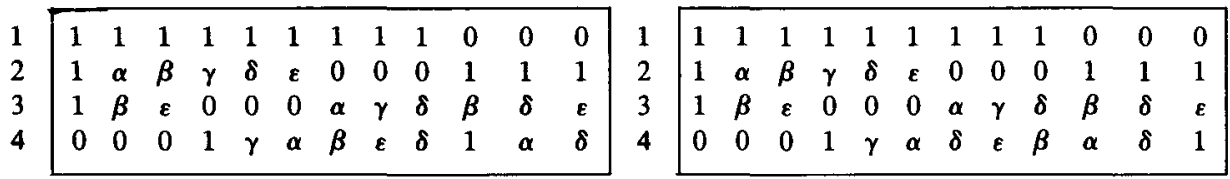


$\begin{array}{llllllllllllllllllllllllll}G_{19} & 1 & 2 & 3 & 1 & 2 & 3 & 1 & 2 & 3 & 1 & 2 & 3 & G_{20} & 1 & 2 & 3 & 4 & 5 & 6 & 7 & 8 & 9 & 10 & 11 & 12\end{array}$

\begin{tabular}{l|llllllllllll|l|lllllllllllll|}
1 & 1 & 1 & 1 & 1 & 1 & 1 & 1 & 1 & 1 & 0 & 0 & 0 & 1 & 1 & 1 & 1 & 1 & 1 & 1 & 1 & 1 & 1 & 0 & 0 & 0 \\
1 & 1 & $\alpha$ & $\beta$ & $\varepsilon$ & $\delta$ & $\gamma$ & 0 & 0 & 0 & 1 & 1 & 1 \\
1 & $\beta$ & $\varepsilon$ & 0 & 0 & 0 & $\delta$ & $\gamma$ & $\alpha$ & $\delta$ & $\beta$ & $\varepsilon$ & & 1 & $\alpha$ & $\beta$ & $\gamma$ & $\delta$ & $\varepsilon$ & 0 & 0 & 0 & 1 & 1 & 1 \\
1 & 0 & 0 & 0 & $\gamma$ & $\delta$ & 1 & $\alpha$ & $\varepsilon$ & $\beta$ & $\beta$ & $\alpha$ & $\varepsilon$ & $\beta$ & $\varepsilon$ & 0 & 0 & 0 & $\alpha$ & $\gamma$ & $\delta$ & $\beta$ & $\delta$ & $\varepsilon$ \\
0 & 0 & 0 & 1 & $\gamma$ & $\alpha$ & $\delta$ & $\beta$ & $\varepsilon$ & 1 & $\delta$ & $\alpha$ \\
\hline
\end{tabular}

$\begin{array}{llllllllllllllllllllllllll}G_{21} & 1 & 2 & 3 & 1 & 2 & 3 & 4 & 5 & 6 & 4 & 5 & 6 & G_{22} & 1 & 2 & 3 & 1 & 2 & 3 & 1 & 2 & 3 & 1 & 2 & 3\end{array}$

\begin{tabular}{lllllllllllll|l|llllllllllll|}
1 & 1 & 1 & 1 & 1 & 1 & 1 & 1 & 1 & 1 & 0 & 0 & 0 \\
1 & 1 & $\alpha$ & $\beta$ & $\varepsilon$ & $\delta$ & $\gamma$ & 0 & 0 & 0 & 1 & 1 & 1 & 1 \\
2 & 1 & $\beta$ & $\varepsilon$ & 0 & 0 & 0 & $\alpha$ & $\gamma$ & $\delta$ & $\delta$ & $\beta$ & $\varepsilon$ \\
0 & 0 & 0 & $\gamma$ & $\delta$ & 1 & $\alpha$ & $\varepsilon$ & $\beta$ & $\varepsilon$ & $\alpha$ & $\beta$ & 1 & 1 & 1 & 1 & 1 & 1 & 1 & 1 & 1 & 0 & 0 & 0 \\
1 & $\alpha$ & $\gamma$ & $\varepsilon$ & $\beta$ & $\delta$ & 0 & 0 & 0 & 1 & 1 & 1 \\
1 & $\gamma$ & $\alpha$ & 0 & 0 & 0 & $\varepsilon$ & $\delta$ & $\beta$ & $\varepsilon$ & $\gamma$ & $\alpha$ \\
0 & 0 & 0 & $\beta$ & 1 & $\delta$ & $\gamma$ & $\varepsilon$ & $\alpha$ & $\beta$ & $\varepsilon$ & $\alpha$ \\
\hline
\end{tabular}

8. $G B R D\left(4,3,6 ; S_{3}\right)$ 's

$\begin{array}{lllllllllllllllllllllllllll}H_{1} & 1 & 1 & 1 & 1 & 1 & 1 & 1 & 1 & 1 & 1 & 1 & 1 & H_{2} & 1 & 1 & 1 & 1 & 1 & 1 & 1 & 1 & 1 & 2 & 2 & 2\end{array}$

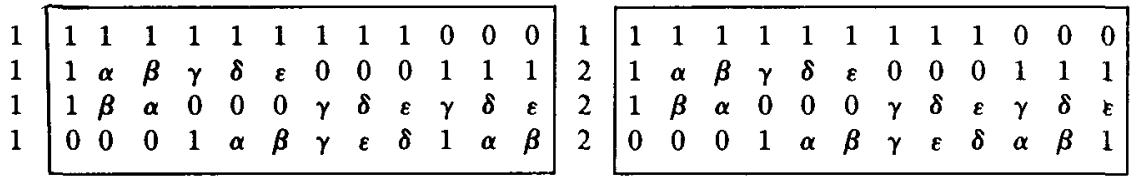

$\begin{array}{lllllllllllllllllllllllllll}H_{3} & 1 & 2 & 1 & 3 & 4 & 3 & 3 & 4 & 3 & 3 & 4 & 3 & H_{4} & 1 & 1 & 1 & 2 & 3 & 4 & 2 & 3 & 4 & 2 & 3 & 4\end{array}$

\begin{tabular}{lllllllllllll|l|llllllllllll|}
1 & 1 & 1 & 1 & 1 & 1 & 1 & 1 & 1 & 1 & 0 & 0 & 0 & 1 & 1 & 1 & 1 & 1 & 1 & 1 & 1 & 1 & 1 & 0 & 0 & 0 \\
1 & $\alpha$ & $\beta$ & $\gamma$ & $\varepsilon$ & $\delta$ & 0 & 0 & 0 & 1 & 1 & 1 & 1 & $\alpha$ & $\beta$ & $\gamma$ & $\delta$ & $\varepsilon$ & 0 & 0 & 0 & 1 & 1 & 1 \\
1 & $\beta$ & $\alpha$ & 0 & 0 & 0 & $\delta$ & $\gamma$ & $\varepsilon$ & $\gamma$ & $\delta$ & $\varepsilon$ & 1 & $\alpha$ & $\beta$ & $\alpha$ & 0 & 0 & 0 & $\varepsilon$ & $\gamma$ & $\delta$ & $\gamma$ & $\delta$ & $\varepsilon$ \\
0 & 0 & 0 & 1 & $\gamma$ & $\alpha$ & $\varepsilon$ & $\beta$ & $\delta$ & 1 & $\delta$ & $\beta$ & 2 & 0 & 0 & 1 & $\alpha$ & $\gamma$ & $\varepsilon$ & $\delta$ & $\beta$ & $\beta$ & 1 & $\delta$ \\
0 & & & & & & & & & & & &
\end{tabular}

$\begin{array}{llllllllllllllllllllllllll}H_{5} & 1 & 2 & 1 & 1 & 2 & 1 & 1 & 2 & 1 & 1 & 2 & 1 & H_{6} & 1 & 1 & 2 & 3 & 4 & 5 & 6 & 7 & 8 & 6 & 7 & 8\end{array}$

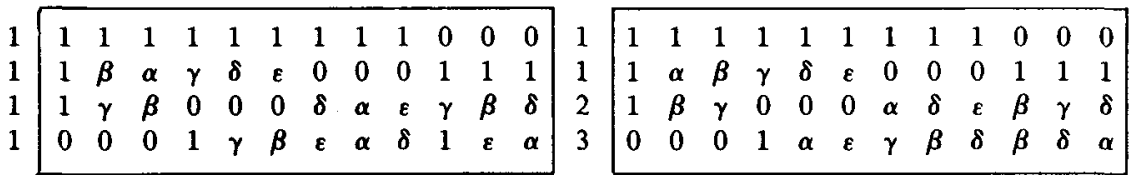

$\begin{array}{llllllllllllllllllllllllll}H_{7} & 1 & 1 & 1 & 2 & 3 & 4 & 2 & 3 & 4 & 2 & 3 & 4 & H_{8} & 1 & 2 & 3 & 4 & 5 & 6 & 7 & 8 & 9 & 10 & 11 & 12\end{array}$

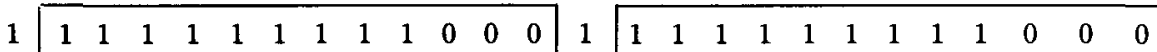

$1 \quad$\begin{tabular}{llllllllllll|llllllllllllll} 
& $\alpha$ & $\gamma$ & $\beta$ & $\delta$ & $\varepsilon$ & 0 & 0 & 0 & 1 & 1 & 1 & 2 & 1 & $\alpha$ & $\gamma$ & $\beta$ & $\delta$ & $\varepsilon$ & 0 & 0 & 0 & 1 & 1 & 1
\end{tabular}

\begin{tabular}{lllllllllllll|l|llllllllllll}
1 & 1 & $\gamma$ & $\beta$ & 0 & 0 & 0 & $\alpha$ & $\varepsilon$ & $\delta$ & $\gamma$ & $\alpha$ & $\beta$ & 3 & 1 & $\gamma$ & $\beta$ & 0 & 0 & 0 & $\alpha$ & $\delta$ & $\varepsilon$ & $\alpha$ & $\beta$ & $\gamma$
\end{tabular}

\begin{tabular}{lllllllllllll|l|llllllllllll}
2 & 0 & 0 & 0 & 1 & $\alpha$ & $\delta$ & $\varepsilon$ & $\beta$ & $\gamma$ & $\gamma$ & $\delta$ & 1 & 4 & 0 & 0 & 0 & 1 & $\alpha$ & $\delta$ & $\gamma$ & $\beta$ & $\varepsilon$ & 1 & $\gamma$ & $\delta$
\end{tabular}

9. $\operatorname{GBRD}\left(5,4,3 ; Z_{3}\right)$

\begin{tabular}{|c|c|c|c|c|c|}
\hline$A$ & 1 & I & 1 & 1 & \\
\hline & 1 & $\boldsymbol{\omega}$ & $\omega$ & 1 & 0 \\
\hline & $\omega$ & $\omega$ & 1 & 0 & 1 \\
\hline & $\boldsymbol{\omega}$ & 1 & 0 & 1 & $\omega$ \\
\hline & 1 & 0 & 1 & $\omega$ & $\omega$ \\
\hline & 0 & 1 & $\boldsymbol{\omega}$ & $\omega$ & 1 \\
\hline
\end{tabular}


10. $G B R D\left(5,4,6 ; Z_{3}\right)$ 's

\begin{tabular}{|c|c|c|c|c|c|c|c|c|c|c|c|c|c|c|c|c|c|c|c|c|c|}
\hline$\beta_{1}$ & 1 & 1 & & & & & & & & & $B_{2}$ & & & & & & & & & & \\
\hline 1 & 1 & $\omega$ & $\omega$ & 1 & 0 & 1 & $\boldsymbol{\omega}$ & $\boldsymbol{\omega}$ & 1 & & 1 & 1 & $\omega$ & $\omega$ & 1 & 0 & 1 & $\bar{\omega}$ & $\bar{\omega}$ & 1 & 0 \\
\hline 1 & $\boldsymbol{\omega}$ & $\boldsymbol{\omega}$ & 1 & 0 & 1 & $\boldsymbol{\omega}$ & $\omega$ & 1 & 0 & 1 & 1 & $\omega$ & $\boldsymbol{\omega}$ & 1 & 0 & 1 & $\bar{\omega}$ & $\bar{\omega}$ & 1 & 0 & 1 \\
\hline 1 & $\omega$ & 1 & 0 & 1 & $\omega$ & $\omega$ & 1 & 0 & 1 & $\omega$ & 1 & $\omega$ & 1 & 0 & 1 & $\omega$ & $\overline{\boldsymbol{\omega}}$ & 1 & 0 & 1 & $\bar{\omega}$ \\
\hline 1 & 1 & 0 & 1 & $\omega$ & $\omega$ & 1 & 0 & 1 & $\boldsymbol{\omega}$ & $\omega$ & 1 & 1 & 0 & 1 & $\boldsymbol{\omega}$ & $\omega$ & 1 & 0 & 1 & $\bar{\omega}$ & $\bar{\omega}$ \\
\hline 1 & 0 & 1 & $\omega$ & $\omega$ & 1 & 0 & 1 & $\omega$ & $\boldsymbol{\omega}$ & 1 & 1 & 0 & 1 & $\omega$ & $\omega$ & 1 & 0 & 1 & $\bar{\omega}$ & $\bar{\omega}$ & 1 \\
\hline$B_{3}$ & 1 & 2 & 2 & 2 & 2 & 1 & 2 & 2 & 2 & 2 & $B_{4}$ & 1 & 2 & 2 & 2 & 2 & 1 & 2 & 2 & 2 & 2 \\
\hline 1 & 1 & $\omega$ & 1 & $\bar{\omega}$ & 0 & 1 & 1 & $\overrightarrow{\boldsymbol{\omega}}$ & $\boldsymbol{\omega}$ & 0 & 1 & 1 & 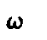 & 1 & & 0 & & $\omega$ & $\bar{\omega}$ & 1 & 0 \\
\hline 1 & 1 & 1 & $\boldsymbol{\omega}$ & 0 & $\bar{\omega}$ & 1 & $\overline{\boldsymbol{\omega}}$ & 1 & 0 & $\omega$ & 1 & 1 & 1 & $\omega$ & 0 & $\overline{\boldsymbol{\omega}}$ & 1 & $\overline{\boldsymbol{\omega}}$ & $\boldsymbol{\omega}$ & 0 & 1 \\
\hline 1 & 1 & $\bar{\omega}$ & 0 & $\omega$ & 1 & 1 & $\omega$ & 0 & 1 & $\overline{\boldsymbol{\omega}}$ & 1 & 1 & $\overline{\boldsymbol{\omega}}$ & 0 & $\omega$ & 1 & 1 & 1 & 0 & $\omega$ & $\overline{\boldsymbol{\omega}}$ \\
\hline 1 & 1 & 0 & $\overline{\boldsymbol{\omega}}$ & 1 & $\omega$ & 1 & 0 & $\omega$ & $\overline{\boldsymbol{\omega}}$ & 1 & 1 & 1 & 0 & $\overline{\boldsymbol{\omega}}$ & 1 & $\boldsymbol{\omega}$ & 1 & 0 & 1 & $\overline{\boldsymbol{\omega}}$ & $\boldsymbol{\omega}$ \\
\hline 2 & 0 & 1 & 1 & 1 & 1 & 0 & 1 & 1 & 1 & 1 & & 0 & 1 & 1 & 1 & 1 & 0 & 1 & 1 & 1 & 1 \\
\hline
\end{tabular}

$\begin{array}{llllllllllllllllllllll}B_{5} & 1 & 2 & 2 & 2 & 2 & 1 & 2 & 2 & 2 & 2 & B_{6} & 1 & 1 & 2 & 2 & 2 & 1 & 1 & 2 & 2 & 2\end{array}$

\begin{tabular}{l|lllll|lllll|l|lllll|lllll|}
1 & 1 & $\bar{\omega}$ & 1 & $\omega$ & 0 & $\omega$ & 1 & $\omega$ & $\bar{\omega}$ & 0 & 1 & 1 & $\omega$ & $\omega$ & 1 & 0 & 1 & $\omega$ & $\omega$ & 1 & 0 \\
1 & 1 & 1 & $\bar{\omega}$ & 0 & $\omega$ & $\omega$ & $\omega$ & 1 & 0 & $\bar{\omega}$ & 1 & $\omega$ & $\omega$ & 1 & 0 & 1 & $\omega$ & $\omega$ & 1 & 0 & 1 \\
1 & $\omega$ & $\bar{\omega}$ & 0 & 1 & $\omega$ & 1 & $\omega$ & 0 & $\bar{\omega}$ & 1 & 1 & $\omega$ & 1 & 0 & 1 & $\omega$ & $\omega$ & 1 & 0 & 1 & $\omega$ \\
1 & $\omega$ & 0 & $\bar{\omega}$ & $\omega$ & 1 & 1 & 0 & $\omega$ & 1 & $\bar{\omega}$ & 2 & 0 & 1 & $\omega$ & $\omega$ & $\bar{\omega}$ & 0 & $\bar{\omega}$ & 1 & 1 \\
2 & 0 & 1 & 1 & 1 & 1 & 0 & 1 & 1 & 1 & 1 & 2 & 1 & $\omega$ & $\omega$ & 1 & 0 & $\bar{\omega}$ & 1 & 1 & $\bar{\omega}$ \\
\hline
\end{tabular}

$\begin{array}{llllllllllllllllllllll}B_{7} & 1 & 1 & 2 & 2 & 2 & 1 & 1 & 2 & 2 & 2 & B_{8} & 1 & 2 & 2 & 3 & 3 & 1 & 2 & 2 & 3 & 3\end{array}$

\begin{tabular}{l|lllll|lllll|l|llllll|lllll|}
1 & 1 & $\omega$ & $\omega$ & 1 & 0 & 1 & $\omega$ & $\omega$ & 1 & 0 & 1 & $\omega$ & $\omega$ & $\bar{\omega}$ & 1 & 0 & $\bar{\omega}$ & $\bar{\omega}$ & $\omega$ & 1 & 0 \\
1 & $\omega$ & $\omega$ & 1 & 0 & 1 & $\omega$ & $\omega$ & 1 & 0 & 1 & 1 & $\omega$ & $\bar{\omega}$ & $\omega$ & 0 & 1 & $\bar{\omega}$ & $\omega$ & $\bar{\omega}$ & 0 & 1 \\
1 & $\omega$ & 1 & 0 & 1 & $\omega$ & $\omega$ & 1 & 0 & 1 & $\omega$ & 2 & 1 & $\bar{\omega}$ & 0 & 1 & $\omega$ & 1 & $\omega$ & 0 & 1 & $\bar{\omega}$ \\
2 & 1 & 0 & 1 & $\omega$ & $\omega$ & $\bar{\omega}$ & 0 & $\bar{\omega}$ & 1 & 1 & 2 & 1 & 0 & $\bar{\omega}$ & $\omega$ & 1 & 1 & 0 & $\omega$ & $\bar{\omega}$ & 1 \\
2 & 0 & 1 & $\omega$ & $\omega$ & 1 & 0 & $\omega$ & $\bar{\omega}$ & $\bar{\omega}$ & $\omega$ & 3 & 0 & 1 & 1 & 1 & 1 & 0 & 1 & 1 & 1 & 1 \\
\hline
\end{tabular}

\begin{tabular}{l|llllllllll}
$B_{9}$ & 1 & 1 & 2 & 2 & 2 & 2 & 2 & 2 & 2 & 2 \\
1 & 1 & 1 & 1 & 1 & $\omega$ & $\bar{\omega}$ & $\omega$ & $\bar{\omega}$ & 0 & 0 \\
1 & 1 & 1 & $\omega$ & $\bar{\omega}$ & 1 & 1 & 0 & 0 & $\omega$ & $\bar{\omega}$ \\
1 & 1 & 1 & $\bar{\omega}$ & $\omega$ & 0 & 0 & $\bar{\omega}$ & $\omega$ & 1 & 1 \\
1 & 1 & 1 & 0 & 0 & $\bar{\omega}$ & $\omega$ & 1 & 1 & $\bar{\omega}$ & $\omega$ \\
2 & 0 & 0 & 1 & 1 & 1 & 1 & 1 & 1 & 1 & 1 \\
\hline
\end{tabular}

11. $G B R D\left(5,4,6 ; Z_{2}\right)$

\begin{tabular}{l|cccccccccc|}
$C$ & 1 & 2 & 1 & 2 & 1 & 2 & 1 & 2 & 1 & 2 \\
\cline { 3 - 8 } \\
1 & + & + & + & + & - & + & + & - & 0 & 0 \\
1 & + & + & - & + & + & - & 0 & 0 & + & + \\
1 & - & + & + & - & 0 & 0 & + & + & + & + \\
1 & + & - & 0 & 0 & + & + & + & + & - & + \\
1 & 0 & 0 & + & + & + & + & - & + & + & - \\
\hline
\end{tabular}

12. $G B R D\left(5,4,6 ; S_{3}\right)$

$$
\begin{array}{lllllllllll}
D & 1 & 2 & 1 & 2 & 1 & 2 & 1 & 2 & 1 & 2
\end{array}
$$

\begin{tabular}{l|llllllllll|}
1 & 1 & 1 & 1 & $\alpha$ & $\gamma$ & 1 & $\beta$ & $\delta$ & 0 & 0 \\
1 & 1 & $\alpha$ & $\gamma$ & 1 & $\beta$ & $\delta$ & 0 & 0 & 1 & 1 \\
1 & $\gamma$ & 1 & $\beta$ & $\delta$ & 0 & 0 & 1 & 1 & 1 & $\alpha$ \\
1 & $\beta$ & $\delta$ & 0 & 0 & 1 & 1 & 1 & $\alpha$ & $\gamma$ & 1 \\
1 & 0 & 0 & 1 & 1 & 1 & $\alpha$ & $\gamma$ & 1 & $\beta$ & $\delta$ \\
\hline
\end{tabular}


13. $G B R D\left(6,5,4 ; Z_{2}\right)$

14. $G B R D\left(6,5,4 ; Z_{4}\right)$

\begin{tabular}{|c|c|c|c|c|c|c|c|c|c|c|c|c|}
\hline $\begin{array}{ll}A & 1\end{array}$ & 1 & 1 & 1 & 1 & 1 & $B$ & 1 & 1 & 1 & 1 & 1 & 1 \\
\hline+ & + & - & - & + & 0 & 1 & 1 & $\alpha$ & 1 & $\beta$ & $\gamma$ & 0 \\
\hline+ & - & - & + & 0 & + & 1 & 1 & 1 & $\beta$ & $\gamma$ & 0 & $\alpha$ \\
\hline+ & - & + & 0 & + & - & 1 & 1 & $\beta$ & $\gamma$ & 0 & $\alpha$ & 1 \\
\hline+ & + & 0 & + & - & - & 1 & 1 & $\gamma$ & 0 & $\alpha$ & 1 & $\beta$ \\
\hline+ & 0 & + & - & - & + & 1 & 1 & 0 & $\alpha$ & 1 & $\beta$ & $\gamma$ \\
\hline 0 & + & + & + & + & + & 1 & 0 & 1 & 1 & 1 & 1 & 1 \\
\hline
\end{tabular}

15. $G B R D\left(6,5,8 ; Z_{2} \times Z_{4}\right)$

\begin{tabular}{l|llllllllllll|}
$C$ & 1 & 2 & 1 & 2 & 1 & 2 & 1 & 2 & 1 & 2 & 1 & 2 \\
1 & 1 & 1 & 1 & 1 & 1 & 1 & 1 & 1 & 1 & 1 & 0 & 0 \\
1 & 1 & $\alpha$ & $\beta$ & $\gamma$ & $\lambda$ & $\delta$ & $\varepsilon$ & $\kappa$ & 0 & 0 & 1 & 1 \\
1 & 1 & $\beta$ & $\varepsilon$ & $\lambda$ & $\kappa$ & $\alpha$ & 0 & 0 & $\delta$ & $\gamma$ & $\kappa$ & $\beta$ \\
1 & 1 & $\gamma$ & $\lambda$ & $\kappa$ & 0 & 0 & $\delta$ & $\beta$ & $\alpha$ & $\varepsilon$ & $\alpha$ & $\kappa$ \\
1 & 1 & $\delta$ & 0 & 0 & $\gamma$ & $\beta$ & $\kappa$ & $\varepsilon$ & $\lambda$ & $\alpha$ & $\beta$ & $\varepsilon$ \\
1 & 0 & 0 & 1 & $\varepsilon$ & $\gamma$ & $\alpha$ & $\delta$ & $\kappa$ & $\beta$ & $\lambda$ & $\lambda$ & $\alpha$ \\
\hline
\end{tabular}

16. $G B R D\left(6,5,8 ; Z_{2}^{3}\right)$ 's

\begin{tabular}{|c|c|c|c|c|c|c|c|c|c|c|c|c|c|c|c|c|c|c|c|c|c|c|c|c|}
\hline$D_{1} 1$ & 1 & 1 & & & & 1 & 1 & & 1 & 1 & 1 & $D_{2}$ & ] & 2 & 1 & 2 & 1 & 2 & 1 & 2 & 1 & & & 2 \\
\hline 1 & $\begin{array}{ll}1 & 1\end{array}$ & 1 & 1 & 1 & 1 & 1 & 1 & 1 & 1 & 0 & 0 & 1 & 1 & 1 & 1 & 1 & 1 & 1 & 1 & 1 & 1 & 1 & 0 & 0 \\
\hline 1 & $1 a$ & $\beta$ & $\gamma$ & $\delta$ & $\boldsymbol{\kappa}$ & $\varepsilon$ & $\lambda$ & 0 & 0 & 1 & 1 & 1 & 1 & $\alpha$ & $\beta$ & $\gamma$ & $\delta$ & $\kappa$ & $\varepsilon$ & $\lambda$ & 0 & 0 & 1 & 1 \\
\hline 1 & $1 \beta$ & $\delta$ & $\dot{\lambda}$ & $\boldsymbol{\varepsilon}$ & $\alpha$ & 0 & 0 & $\gamma$ & $\kappa$ & $\beta$ & $\boldsymbol{E}$ & 1 & 1 & $\beta$ & $\delta$ & $\dot{\lambda}$ & $\varepsilon$ & $\gamma$ & 0 & 0 & $\alpha$ & $\kappa$ & $\lambda$ & $\beta$ \\
\hline 1 & $1 \gamma$ & $\lambda$ & $\varepsilon$ & 0 & 0 & $\alpha$ & $\boldsymbol{\kappa}$ & $\delta$ & $\boldsymbol{\beta}$ & $\lambda$ & $\gamma$ & 1 & 1 & $\gamma$ & $\lambda$ & $\varepsilon$ & 0 & 0 & $\alpha$ & $\delta$ & $\kappa$ & $\beta$ & $\alpha$ & $\lambda$ \\
\hline 1 & $1 \kappa$ & 0 & 0 & $\lambda$ & $\delta$ & $\gamma$ & $\beta$ & $\varepsilon$ & $\alpha$ & $\alpha$ & $\delta$ & 1 & 1 & $\delta$ & 0 & 0 & $\beta$ & $\alpha$ & $\lambda$ & $\kappa$ & $\varepsilon$ & $\gamma$ & $\delta$ & $\gamma$ \\
\hline c & $\begin{array}{lll}0 & 0\end{array}$ & 1 & $\varepsilon$ & $\gamma$ & $\beta$ & $\delta$ & $\lambda$ & $\kappa$ & $\alpha$ & $\gamma$ & $\boldsymbol{\varepsilon}$ & 1 & 0 & 0 & 1 & $\lambda$ & $\delta$ & $\boldsymbol{\varepsilon}$ & $\beta$ & $\alpha$ & $\kappa$ & $\gamma$ & $\varepsilon$ & $\alpha$ \\
\hline
\end{tabular}

17. $G B R D(6,5,8 ; Q)$ 's

$E_{1}$\begin{tabular}{llllllllllllllllllllllllll|}
1 & 2 & 1 & 2 & 1 & 2 & 1 & 2 & 1 & 2 & 1 & 2 & $E_{2}$ & 1 & 2 & 1 & 2 & 1 & 2 & 1 & 2 & 1 & 2 & 1 & 2 \\
1 & 1 & 1 & 1 & 1 & 1 & 1 & 1 & 1 & 1 & 1 & 0 & 0 & 1 & 1 & 1 & 1 & 1 & 1 & 1 & 1 & 1 & 1 & 1 & 0 & 0 \\
1 & 1 & $\alpha$ & $\gamma$ & $\beta$ & $\delta$ & $\kappa$ & $\lambda$ & $\varepsilon$ & 0 & 0 & 1 & 1 & 1 & 1 & $\alpha$ & $\gamma$ & $\beta$ & $\delta$ & $\kappa$ & $\lambda$ & $\varepsilon$ & 0 & 0 & 1 & 1 \\
1 & 1 & $\beta$ & $\lambda$ & $\gamma$ & $\varepsilon$ & $\delta$ & 0 & 0 & $\kappa$ & $\alpha$ & $\gamma$ & $\beta$ & 1 & 1 & $\beta$ & $\alpha$ & $\kappa$ & $\varepsilon$ & $\lambda$ & 0 & 0 & $\delta$ & $\gamma$ & $\gamma$ & $\beta$ \\
1 & 1 & $\varepsilon$ & $\kappa$ & $\alpha$ & 0 & 0 & $\delta$ & $\lambda$ & $\beta$ & $\gamma$ & $\kappa$ & $\alpha$ & 1 & 1 & $\gamma$ & $\lambda$ & $\alpha$ & 0 & 0 & $\varepsilon$ & $\delta$ & $\kappa$ & $\beta$ & $\lambda$ & $\alpha$ \\
1 & 1 & $\gamma$ & 0 & 0 & $\beta$ & $\varepsilon$ & $\kappa$ & $\delta$ & $\alpha$ & $\lambda$ & $\lambda$ & $\delta$ & 1 & 1 & $\delta$ & 0 & 0 & $\beta$ & $\varepsilon$ & $\gamma$ & $\kappa$ & $\alpha$ & $\lambda$ & $\beta$ & $\varepsilon$ \\
1 & 0 & 0 & $\beta$ & 1 & $\alpha$ & $\varepsilon$ & $\lambda$ & $\kappa$ & $\delta$ & $\gamma$ & $\delta$ & $\beta$ & 1 & 0 & 0 & $\lambda$ & 1 & $\kappa$ & $\gamma$ & $\beta$ & $\varepsilon$ & $\alpha$ & $\delta$ & $\gamma$ & $\lambda$ \\
\hline
\end{tabular}


A3. Contracted $G B R D(k+1, k, l(k-1) ; G)$ 's

1. $G B R D\left(6,5,8 ; Z_{4}\right)$

\begin{tabular}{l|llllllllllll|}
$\psi$ & 1 & 2 & 1 & 2 & 1 & 2 & 1 & 2 & 1 & 2 & 1 & 2 \\
1 & 1 & 1 & 1 & 1 & 1 & 1 & 1 & 1 & 1 & 1 & 0 & 0 \\
1 & 1 & $\alpha$ & $\beta$ & $\gamma$ & $\gamma$ & 1 & $\alpha$ & $\beta$ & 0 & 0 & 1 & 1 \\
1 & 1 & $\beta$ & $\alpha$ & $\gamma$ & $\beta$ & $\alpha$ & 0 & 0 & 1 & $\gamma$ & $\beta$ & $\beta$ \\
1 & 1 & $\gamma$ & $\gamma$ & $\beta$ & 0 & 0 & 1 & $\beta$ & $\alpha$ & $\alpha$ & $\alpha$ & $\beta$ \\
1 & 1 & 1 & 0 & 0 & $\gamma$ & $\beta$ & $\beta$ & $\alpha$ & $\gamma$ & $\alpha$ & $\beta$ & $\alpha$ \\
1 & 0 & 0 & 1 & $\alpha$ & $\gamma$ & $\alpha$ & 1 & $\beta$ & $\beta$ & $\gamma$ & $\gamma$ & $\alpha$ \\
\hline
\end{tabular}

2. $G B R D\left(6,5,8 ; Z_{2}^{2}\right)$ 's

$\begin{array}{llllllllllllllllllllllllll}\Phi_{1} & 1 & 2 & 1 & 2 & 1 & 2 & 1 & 2 & 1 & 2 & 1 & 2 & \Phi_{2} & 1 & 1 & 1 & 1 & 2 & 3 & 2 & 3 & 2 & 3 & 2 & 3\end{array}$

\begin{tabular}{lllllllllllll|l|llllllllllll|}
1 & 1 & 1 & 1 & 1 & 1 & 1 & 1 & 1 & 1 & 1 & 0 & 0 \\
1 & 1 & $\alpha$ & 1 & $\alpha$ & $\gamma$ & $\beta$ & $\gamma$ & $\beta$ & 0 & 0 & 1 & 1 & 1 & 1 & 1 & 1 & 1 & 1 & 1 & 1 & 1 & 1 & 1 & 0 & 0 \\
1 & 1 & 1 & $\alpha$ & $\alpha$ & $\beta$ & $\gamma$ & $\beta$ & $\gamma$ & 0 & 0 & 1 & 1 \\
1 & 1 & $\gamma$ & $\gamma$ & $\beta$ & $\alpha$ & 0 & 0 & $\beta$ & $\alpha$ & $\beta$ & 1 \\
1 & 1 & $\alpha$ & $\gamma$ & $\beta$ & 0 & 0 & $\beta$ & 1 & $\alpha$ & $\gamma$ & $\alpha$ & $\beta$ & $\alpha$ & $\beta$ & $\gamma$ & $\beta$ & 1 & 0 & 0 & $\alpha$ & $\gamma$ & $\beta$ & $\alpha$ \\
1 & 1 & $\beta$ & 0 & 0 & $\alpha$ & 1 & $\beta$ & $\gamma$ & $\gamma$ & $\alpha$ & 1 & $\gamma$ & $\alpha$ & $\alpha$ & $\gamma$ & $\beta$ & 0 & 0 & 1 & $\gamma$ & $\beta$ & $\alpha$ & $\alpha$ & $\gamma$ \\
1 & 0 & 0 & 1 & $\gamma$ & $\alpha$ & $\alpha$ & $\beta$ & $\beta$ & 1 & $\gamma$ & $\gamma$ & $\alpha$ & 2 & $\gamma$ & 0 & 0 & $\gamma$ & $\beta$ & $\alpha$ & $\alpha$ & $\beta$ & 1 & $\beta$ & 1 \\
0 & 0 & 1 & $\beta$ & $\alpha$ & $\alpha$ & $\beta$ & $\gamma$ & $\gamma$ & 1 & $\beta$ & $\alpha$ \\
\hline
\end{tabular}

$\begin{array}{llllllllllllllllllllllllll}\Phi_{3} & 1 & 2 & 1 & 2 & 1 & 2 & 1 & 2 & 1 & 2 & 1 & 2 & \Phi_{4} & 1 & 2 & 1 & 2 & 3 & 4 & 3 & 4 & 3 & 4 & 3 & 4\end{array}$

\begin{tabular}{|c|c|c|c|c|c|c|c|c|c|c|c|c|c|c|c|c|c|c|c|c|c|c|c|}
\hline & 1 & 1 & 1 & 1 & 1 & 1 & 1 & 1 & 0 & 0 & 1 & 1 & 1 & 1 & 1 & 1 & 1 & 1 & 1 & 1 & 1 & 0 & 0 \\
\hline$\alpha$ & $\gamma$ & $\beta$ & 1 & $\beta$ & $\gamma$ & $\alpha$ & 0 & 0 & 1 & 1 & 1 & 1 & 1 & $\alpha$ & $\alpha$ & $\beta$ & $\gamma$ & $\beta$ & $\gamma$ & 0 & 0 & 1 & 1 \\
\hline$\beta$ & $\gamma$ & 1 & $\alpha$ & $\alpha$ & 0 & 0 & $\beta$ & $\gamma$ & $\alpha$ & $\beta$ & 1 & 1 & $\alpha$ & $\beta$ & $\gamma$ & $\beta$ & $\alpha$ & 0 & 0 & 1 & $\gamma$ & $\gamma$ & $\alpha$ \\
\hline$\gamma$ & $\alpha$ & $\gamma$ & 0 & 0 & $\beta$ & $\alpha$ & $\beta$ & 1 & $\gamma$ & $\gamma$ & 1 & 1 & $\alpha$ & $\gamma$ & $\beta$ & 0 & 0 & 1 & $\beta$ & $\gamma$ & $\alpha$ & 1 & $\gamma$ \\
\hline$\beta$ & 0 & 0 & $\gamma$ & 1 & $\beta$ & $\gamma$ & $\alpha$ & $\alpha$ & 1 & $\alpha$ & 2 & 1 & $\beta$ & 0 & 0 & $\alpha$ & 1 & $\gamma$ & $\gamma$ & $\beta$ & $\alpha$ & $\beta$ & $\alpha$ \\
\hline 0 & $\alpha$ & 1 & $\gamma$ & $\beta$ & $\gamma$ & 1 & $\alpha$ & $\beta$ & $\alpha$ & $\gamma$ & 2 & 0 & 0 & 1 & $\gamma$ & $\beta$ & $\beta$ & $\alpha$ & 1 & $\gamma$ & $\alpha$ & $\beta$ & 1 \\
\hline
\end{tabular}

3. $\operatorname{GBRD}\left(6,5,8 ; Z_{2}\right)$ 's

$\begin{array}{llllllllllllllllllllllllll}\Omega_{1} & 1 & 1 & 1 & 1 & 1 & 1 & 1 & 1 & 1 & 1 & 1 & 1 & \Omega_{2} & 1 & 2 & 1 & 2 & 1 & 2 & 1 & 2 & 1 & 2 & 1 & 2\end{array}$

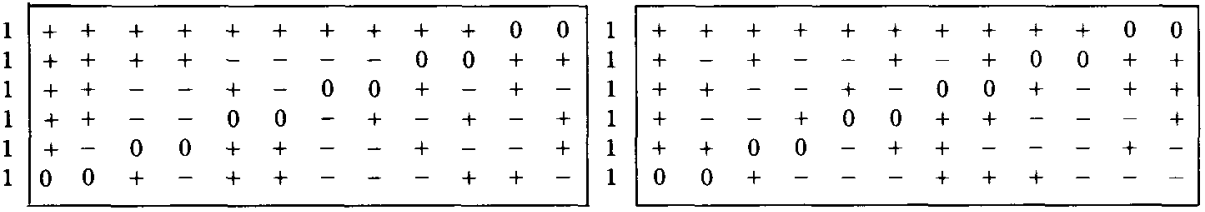

$\begin{array}{llllllllllllllllllllllllll}\Omega_{3} & 1 & 1 & 1 & 1 & 1 & 1 & 1 & 1 & 1 & 1 & 1 & 1 & \Omega_{4} & 1 & 1 & 1 & 1 & 1 & 1 & 1 & 1 & 1 & 1 & 1 & 1\end{array}$

\begin{tabular}{|c|c|c|c|c|c|c|c|c|c|c|c|c|c|c|c|c|c|c|c|c|c|c|c|c|}
\hline+ & + & - & - & + & 0 & - & + & + & - & + & 0 & 1 & + & + & - & - & + & 0 & + & - & - & - & + & 0 \\
\hline+ & - & - & + & 0 & + & + & + & - & - & 0 & + & 1 & + & $\rightarrow$ & - & + & 0 & + & + & - & + & + & 0 & + \\
\hline+ & - & + & 0 & + & - & + & + & + & 0 & - & - & 1 & + & - & + & 0 & + & - & - & - & + & 0 & + & - \\
\hline+ & + & 0 & + & - & - & - & + & 0 & + & - & + & 1 & + & + & 0 & + & - & - & + & + & 0 & + & + & - \\
\hline+ & 0 & + & - & - & + & + & 0 & + & + & + & + & 1 & + & 0 & + & - & - & + & + & 0 & + & - & - & - \\
\hline 0 & + & + & + & + & + & 0 & + & - & + & + & - & 1 & 0 & + & + & + & + & + & 0 & + & + & - & + & + \\
\hline
\end{tabular}




\section{References}

[1] M. Bhaskar Rao, 'A group divisible family of PBIB designs, J. Indian Stat. Association 4 (1966), 14-28.

[2] A. T. Butson, 'Generalized Hadamard matrices', Proc. Amer. Math. Soc. 13 (1962), 894-898.

[3] A. T. Butson, 'Relations among generalized Hadamard matrices, relative difference sets and maximal length recurring sequences', Canad. J. Math. 15 (1963), 42-48.

[4] C. J. Colbourn and S. A. Vanstone, 'Doubly resolvable twofold triple systems', Congressus Numerantium 34 (1982), 219-223.

[5] P. Delsarte and J. M. Goethals, 'On quadratic residue-like sequences in Abelian groups', Report R168, MBLE Research Laboratory, Brussels (1971).

[6] P. Delsarte and J. M. Goethals, 'Tri-weight codes and generalized Hadamard matrices', Information and Control 15 (1969), 196-206.

[7] D. A. Drake, 'Partial $\lambda$-geometries and generalized Hadamard matrices over groups', Canad. $J$. Math. 31 (1979), 617-627.

[8] A. V. Geramita, N. J. Pullman and J. Seberry Wallis, 'Families of weighing matrices', Bull. A ustral. Math. Soc. 10 (1974), 119-122.

[9] A. V. Geramita and J. Seberry, Orthogonal designs: quadratic forms and Hadamard matrices (Marcel Dekker, New York, 1979).

[10] P. B. Gibbons, 'Computing techniques for the construction and analysis of block designs', Tech. Report \# 92, Dept. of Computer Science, University of Toronto (May 1976).

[11] P. B. Gibbons, R. Mathon and D. G. Corneil, 'Computing techniques for the construction and analysis of block designs', Utilitas Math. 11 (1977), 161-192.

[12] J. M. Goethals and J. J. Seidel, 'Orthogonal matrices with zero diagonal', Canad. J. Math. 19 (1967), 1001-1010.

[13] R. Hain, Circulant weighing matrices (M. Sc. Thesis, Australian National University, Canberra 1977).

[14] H. D. L. Hollmann, Association schemes (M. Sc. thesis, Eindhoven University of Technology, 1982).

[15] R. Mathon, 'Symmetric conference matrices of order $p q^{2}+1$ ', Canad. J. Math. 30 (1978), 321-331.

[16] R. Mathon, '3-class association schemes' (in Proc. Conf. on Algebraic Aspects of Combinatorics, Toronto 1975, 123-155).

[17] R. A. Mathon, K. T. Phelps and A. Rosa, 'A class of Steiner triple systems of order 21 and associated Kirkman systems', Math. of Comp. 37 (1981), 209-222.

[18] R. Mathon and A. Rosa, 'The 4-rotational Steiner and Kirkman triple systems of order 21', Ars Combinatoria 17A (1984), 241-250.

[19] R. Mathon and S. A. Vanstone, 'On the existence of doubly resolvable Kirkman systems and equidistant permutation arrays', Discrete Math. 30 (1980), 157-172.

[20] R. C. Read, 'Every one a winner', Ann. Discrete Math. 2 (1978), 107-120.

[21] A. Rosa, 'Room squares generalized', Ann. Discrete Math. 8 (1980), $43-57$.

[22] J. Seberrry, 'Some families of partially balanced incomplete block designs' (Combinatorial Mathematics IX, Lecture Notes in Math. 829, Springer, Berlin, 1982).

[23] J. Seberry, 'Some remarks on generalized Hadamard matrices and theorems of Rajkundlia on SBIBD's (Combinatorial Mathematics VI, Lecture Notes in Math. 748, Springer, 1979, 154-164).

[24] S. S. Shrikhande, 'Generalized Hadamard matrices and orthogonal arrays of strength 2', Canad. J. Math. 16 (1964), 736-740.

[25] D. J. Street and C. A. Rodger, 'Some results on Bhaskar Rao designs', (Combinatorial Mathematics VII, edited by R. W. Robinson, C. W. Southern and W. D. Wallis, Lecture Notes in Mathematics, 829, Springer-Verlag, 1980, 238-245). 
[26] S. A. Vanstone and R. C. Mullin, 'A note on existence of weighing matrices $W\left(2^{2 n-j}, 2^{n}\right)$ and associated combinatorial designs', Utilitas Math. 8 (1975), 371-381.

[27] J. Seberry Wallis, 'Hadamard Matrices' (Part IV of Combinatorics: Room sequences, sum free sets and Hadamard matrices, by W. D. Wallis, Anne Penfold Street and Jennifer Seberry Wallis, in Lecture Notes in Mathematics 292, Springer-Verlag, 1972, 273-489).

[28] R. M. Wilson, 'Symmetric group divisible designs', unpublished manuscript.

[29] F. Yates, 'Complex experiments', J. Royal Stat. Soc. B2 (1935), 181-223.

Department of Computer Science

University of Auckland

Auckland

New Zealand
Department of Computer Science

University of Toronto

Toronto, Ontario

Canada (M5S 1A4) 